\title{
XMM-Newton observation of the classical T Tauri star SU Aurigae and the surrounding field ${ }^{\star}$
}

\author{
E. Franciosini ${ }^{1}$, L. Scelsi ${ }^{2}$, R. Pallavicini ${ }^{1}$, and M. Audard ${ }^{3,4}$ \\ 1 INAF - Osservatorio Astronomico di Palermo, Piazza del Parlamento 1, 90134 Palermo, Italy \\ e-mail: francio@astropa.inaf.it \\ 2 Dipartimento di Scienze Fisiche ed Astronomiche, Università di Palermo, Piazza del Parlamento 1, 90134 Palermo, Italy \\ 3 Integral Science Data Centre, Ch. d'Ecogia 16, 1290 Versoix, Switzerland \\ ${ }^{4}$ Geneva Observatory, University of Geneva, Ch. des Maillettes 51, 1290 Sauverny, Switzerland
}

Received 16 January 2007 /Accepted 18 June 2007

\section{ABSTRACT}

\begin{abstract}
Aims. We investigate the properties of the X-ray emitting plasma of the classical T Tauri star SU Aurigae and of other sources in the field of view.

Methods. We use XMM-Newton to obtain a high-resolution RGS spectrum of SU Aur as well as EPIC imaging data and lowresolution spectra of the star and of other X-ray sources in the surrounding field. We reconstruct the emission measure distribution of SU Aur from the RGS spectrum using a line-based method, and we perform multi-temperature fits of the MOS spectra of the strongest sources both for the full observation and for selected time intervals to study their spectral variability.

Results. The emission from SU Aur is highly variable, showing three flares during the observation. The MOS spectra indicate a very hot corona, with significant emissivity up to $\sim 40 \mathrm{MK}$ in quiescence, and temperatures up to $140 \mathrm{MK}$ during flares. The emission measure distribution derived from the RGS spectrum peaks at $\log T=7.0$; any contribution to the X-ray luminosity from cool plasma $(T \sim 2 \mathrm{MK})$ cannot exceed 5\% of the total emission. Abundances are $\sim 0.3-0.6$ solar with the exception of $\mathrm{Mg}$ and $\mathrm{Ne}$ that are solar. Spatial analysis of the full EPIC field results in the detection of 104 X-ray sources, 6 of which are associated with the known Taurus-Auriga members in the field of view (including SU Aur).

Conclusions. The characteristics of the X-ray emission of SU Aur are very similar to those of young active late-type stars, with a very hot corona and flares, suggesting magnetic activity as the origin of most of the X-ray emission, rather than accretion.
\end{abstract}

Key words. stars: activity - stars: coronae - stars: pre-main sequence - stars: late-type - X-rays: stars open clusters and associations: individual: Taurus-Auriga

\section{Introduction}

T Tauri stars are young pre-main sequence (PMS) late-type stars, characterized by the presence of strong X-ray emission (Feigelson \& Montmerle 1999; Favata \& Micela 2003). X-ray emission is commonly observed both from classical T Tauri stars (CTTS), that show strong $\mathrm{H} \alpha$ emission indicative of ongoing accretion from a thick circumstellar disk, and weak-lined T Tauri stars (WTTS), with weaker $\mathrm{H} \alpha$ emission, where active accretion has ended, although they could still be surrounded by circumstellar material.

X-ray emission from CTTS and WTTS is commonly attributed to solar-like magnetic activity, similarly to what observed in older active stars. However a still debated question is whether and to what extent the presence of accretion and/or of a thick circumstellar disk may influence the emission process. Studies of several star-forming regions have shown that the $\mathrm{X}$-ray emission of $\mathrm{T}$ Tauri stars does not appear to be related to the presence or not of a disk, as indicated by infrared excess (e.g. Feigelson et al. 2003; Preibisch et al. 2005), but is affected by active accretion, that reduces significantly the X-ray luminosity of CTTS with respect to WTTS (e.g. Stelzer \& Neuhäuser 2001;

$\star$ Table 2 is only available in electronic form at http://www. aanda.org
Flaccomio et al. 2003; Stassun et al. 2004; Preibisch et al. 2005; Franciosini et al. 2006; Telleschi et al. 2007a).

High-resolution X-ray spectroscopy allows us to better investigate the $\mathrm{X}$-ray emission processes in PMS stars, giving access to detailed plasma diagnostics in terms of temperature structure, elemental abundances and density of the emitting plasma. Chandra and XMM-Newton observations of the CTTS TW Hya have shown that the X-ray emitting material in this star is dominated by cool plasma ( $T \sim 3 \mathrm{MK})$, with a very low $f / i$ line ratio in the $\mathrm{O}$ VII and Ne IX He-like triplets, indicative of very high densities $\left(n_{\mathrm{e}} \gtrsim 10^{12} \mathrm{~cm}^{-3}\right)$ or a strong ultraviolet flux. These characteristics are very different from those found in magnetically active stars, and have been interpreted as emission from an accretion shock due to material falling from the disk onto the stellar surface (Kastner et al. 2002; Stelzer \& Schmitt 2004). Similar low $f / i$ ratios have been found also for the CTTS BP Tau (Schmitt et al. 2005), CR Cha (Robrade \& Schmitt 2006), V4046 Sgr (Günther et al. 2006), and MP Mus (Argiroffi et al. 2007), that however show also the presence of hotter material at $\sim 10-20 \mathrm{MK}$ and flares, suggesting that at least part of the X-ray emission is of magnetic origin. On the other hand, no evidence for high densities was found for the Herbig Ae star AB Aur (Telleschi et al. 2007c) and for the CTTS T Tau (Güdel et al. 2007b). Telleschi et al. (2007b) found an enhanced $\mathrm{O}$ VII $(r) / \mathrm{O}$ VIII $(\mathrm{Ly} \alpha)$ ratio in CTTS compared to WTTS and 
active main-sequence stars, and suggested that the presence of a soft excess emission, rather that high densities, might be a characteristic feature of accreting stars. Telleschi et al. (2007a) and Güdel et al. (2007b) proposed that cool material from accretion streams might penetrate into hotter active regions and reduce the plasma temperature. According to the above authors, this could explain the excess emission at low temperatures seen in the RGS, as well as the lower X-ray luminosity of CTTS with respect to WTTS in the broad X-ray band of CCD instruments, since CCD spectroscopy does not access the softest X-ray components. A similar scenario was proposed by Preibisch et al. (2005), who suggested that magnetic structures loaded with accreting material would be denser and could not be heated to Xray emitting temperatures. A problem with these interpretations is the very small surface filling factors of the accretion streams predicted from accretion models (Calvet \& Gullbring 1998), although higher filling factors are possible if the plasma density is lower. An alternative model considers stripping of the outer parts of the coronal structures due to accretion as responsible for the lower X-ray luminosity of CTTS (Jardine et al. 2006).

SU Aur (G2III, age $\sim 4 \mathrm{Myr}, M=2 M_{\odot}, P_{\text {rot }}=1.76 \mathrm{~d}$; DeWarf et al. 2003) is one of the brightest CTTS at X-ray wavelengths, belonging to the Taurus-Auriga region, one of the nearest ( $d \sim 140 \mathrm{pc}$ ) and most active regions of low-mass star formation. SU Aur is located in the L1517 cloud, and has an Hipparcos distance of $152_{-34}^{+63} \mathrm{pc}$ (ESA 1997). The star was first detected by Einstein with an X-ray luminosity of $\sim 3-4 \times 10^{30} \mathrm{erg} \mathrm{s}^{-1}$ in the 0.5-4.5 keV band (Feigelson \& DeCampli 1981; Damiani et al. 1995). It was later detected in the ROSAT All-Sky Survey with a similar X-ray luminosity; spectral analysis yielded a temperature of $\sim 1.3 \mathrm{keV}$ (Neuhäuser et al. 1995). In a pointed ROSAT/PSPC observation, Wichmann et al. (1996) found $T=1.45 \mathrm{keV}$ and $L_{\mathrm{X}} \sim 6 \times 10^{30} \mathrm{erg} \mathrm{s}^{-1}$. Skinner \& Walter (1998) observed SU Aur with $A S C A$, finding temperatures of 0.8 and $2.5 \mathrm{keV}$ and emission measures of $10^{53}$ and $4 \times 10^{53} \mathrm{~cm}^{-3}$; the X-ray luminosity in the $0.5-10 \mathrm{keV}$ band was $8 \times 10^{30} \mathrm{erg} \mathrm{s}^{-1}$.

SU Aur has been recently observed with Chandra and XMM-Newton. During the Chandra observation a strong flare was observed (Smith et al. 2005). Time-dependent spectroscopy indicated the presence of hot plasma at temperatures of 30-40 MK in quiescence, and rising up to $100 \mathrm{MK}$ during the flare. In this paper we report the results of the XMM-Newton EPIC and RGS observation of SU Aur and of the surrounding region. A partial analysis of this observation has been reported by Robrade \& Schmitt (2006) who compared the emission properties of a sample of CTTS stars, and by Telleschi et al. (2007b) who analyzed the RGS spectra of a sample of WTTS and CTTS in the Taurus molecular cloud. However, the above authors used a global fitting approach to the EPIC and RGS spectra to derive the thermal structure of the stellar corona, while here we adopt a line-based method to reconstruct the emission measure distribution (EMD). The EMD can allow us to gain insights into the properties of the coronal structures and the loop populations involved in the X-ray production (Peres et al. 2001). In fact, being the plasma optically thin, the global EMD can be viewed as the sum of the emission measure distributions of all the looplike structures where the plasma is magnetically confined. It is therefore interesting to compare the EMD of CTTS with those of stars without disks to investigate possible differences. We also present a detailed time-dependent analysis of the X-ray emission of SU Aur as well as an analysis of the entire EPIC field.

The paper is organized as follows. Observation and data analysis are described in Sect. 2. In Sects. 3 and 4 we present the results of the analysis of the EPIC and RGS spectra,

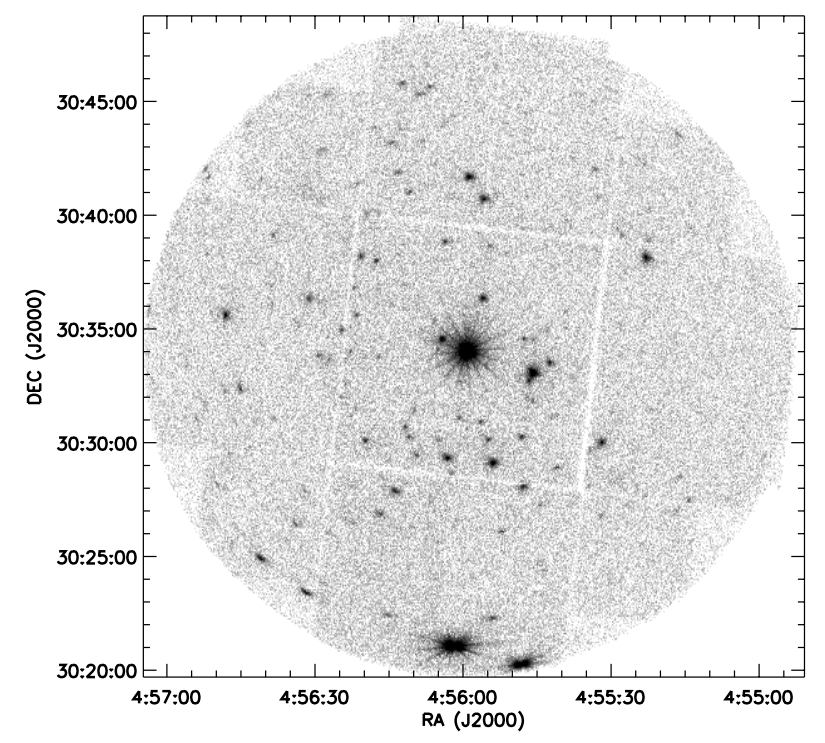

Fig. 1. Combined EPIC MOS1+MOS2 image of the field around SU Aur.

respectively. In Sect. 5 we discuss the detection and analysis of other sources in the field of view. Discussion and conclusions are given in Sect. 6.

\section{Observation and data analysis}

SU Aur was observed by XMM-Newton as part of the GT program of one of us (R.P.), using both the RGS instrument and the EPIC MOS cameras (the PN camera was not operating during the observation). The observation (ID 0101440801) started at 01:28 UT on September 21, 2001 and ended at 13:35 UT on September 22, 2001, for a total duration of $130 \mathrm{ks}$. The EPIC cameras were operated in Full Frame mode using the thick filter. Data processing was carried out using the standard tasks in SAS v.6.1.0. Both RGS and EPIC event files have been time filtered to exclude a few short periods of high background due to proton flares at the beginning and at the end of the observation; the final effective exposure time is $123 \mathrm{ks}$ for each MOS and $118 \mathrm{ks}$ for each RGS.

\subsection{EPIC}

The combined EPIC MOS1+MOS2 image in the $0.3-7.8 \mathrm{keV}$ energy band is shown in Fig. 1. The EPIC field of view contains several bright X-ray sources in addition to SU Aur, which is the brightest source in the centre of the field. We performed a source detection both on the individual and on the merged MOS1+MOS2 datasets in the $0.3-7.8 \mathrm{keV}$ band, using the Wavelet Detection algorithm developed at INAF-Osservatorio Astronomico di Palermo (Damiani et al. 1997), adapted to the EPIC case. This algorithm computes wavelet transforms on different spatial scales, allowing the detection of both pointlike and extended sources. The EPIC version has been specifically designed to handle in a straightforward way source detection on the sum of datasets from different instruments. After removing a few obviously spurious detections due to hot pixels and to the point spread function structure of the central bright source, we obtained a total of 104 sources detected above a significance threshold of $5 \sigma$. To search for systematic offsets in the $\mathrm{X}$-ray positions, we cross-correlated the X-ray source list with the 2MASS catalogue (Skrutskie et al. 2006) adopting as a first 
step a search radius of $6^{\prime \prime}$. We then corrected the X-ray coordinates and repeated the procedure with a $4^{\prime \prime}$ radius. The total median coordinate shift applied to the X-ray positions was $-3.1^{\prime \prime}$ in right ascension and 1.7" in declination.

MOS1 and MOS2 light curves and spectra of SU Aur were extracted from the event files using a circular region of radius $50^{\prime \prime}$ centered on the source. For the other bright source HD 31305 studied in Sect. 5, we used an extraction radius of $24^{\prime \prime}$. Background light curves and spectra were extracted from nearby circular regions free from other X-ray sources and on the same CCD chip, using the same extraction radius as for the corresponding source. The MOS1 and MOS2 spectra were rebinned in order to have at least 20 counts per bin, and have been jointly fitted in XSPEC v.11.3.2, using an absorbed optically thin APEC v.1.3.1 plasma model with three thermal components and variable individual abundances for SU Aur, and two thermal components and variable global abundance for HD 31305. All abundances were computed relative to the solar photospheric abundances by Anders \& Grevesse (1989). Unabsorbed X-ray luminosities in the $0.3-8 \mathrm{keV}$ band have been derived from the best-fit models, using the Taurus-Auriga distance of $140 \mathrm{pc}$. Note that we used this distance instead of the Hipparcos one for SU Aur, for consistency with previous studies and for comparison with the other sources in the field.

\subsection{RGS}

RGS1 and RGS2 source and background spectra of SU Aur were extracted from the event files using the standard extraction regions. We note that the RGS field of view contains also the nearby source AB Aur, located at a distance of 3.1' from SU Aur (DeWarf et al. 2003). AB Aur is 20 times weaker than SU Aur in EPIC (see Table 3), however it displays a weak spectrum in the RGS, that could in principle contaminate the SU Aur spectrum (see Telleschi et al. 2007c, for a detailed analysis of the RGS spectrum of AB Aur). Our choice of the source extraction region does not include a significant contribution from AB Aur, given the separation of the two stars along the cross-dispersion direction, although some contamination might be present in the background spectrum. Any contaminating lines from AB Aur can be easily distinguished, since the $\mathrm{AB}$ Aur spectrum is displaced, with respect to that of SU Aur, by $\sim 0.4 \AA$ due to the relative position of the two stars along the dispersion direction. We have carefully checked the background-subtracted spectrum of SU Aur, finding no evidence for significant contaminating lines or count deficits, compared with the noise fluctuations, at the expected wavelenghts for the strongest lines of AB Aur (see Telleschi et al. 2007c).

\subsubsection{EMD reconstruction}

The RGS1 and RGS2 spectra have been analyzed using PINTofALE (Kashyap \& Drake 2000) to derive the EMD and the abundances from the measurement of individual line fluxes. We used the APED v. 1.3 emission line database and the collisional ionization equilibrium by Mazzotta et al. (1998). As for the EPIC fits, derived abundances are relative to those by Anders $\&$ Grevesse (1989). In all steps of the analysis we have properly taken into account the interstellar absorption for the evaluation of both the continuum and the line fluxes, using the value of the hydrogen column density derived from the MOS fit (see Sect. 3).

Following the procedure described by Scelsi et al. (2004), we added the two background-subtracted RGS spectra together after an appropriate rebinning, to increase the signal-to-noise ratio, and we measured the line fluxes assuming Lorentzian line profiles. In order to obtain reliable line fluxes, an accurate estimate of the continuum level is needed. Unfortunately, the large wings of the RGS line spread function (LSF) make it difficult to identify the true continuum level. To this aim, we therefore used an iterative approach. As a first step, a continuum level was evaluated using the results of the three-temperature MOS spectral fitting and was then adopted in the measurement of the fluxes of all evident lines in the spectrum. We then reconstructed a trial EMD, excluding all lines with low signal-to-noise ratio $(S / N<1.5)$, lines with problems in the combined LSF due to CCD gaps or bad pixels, for which the flux measurement is likely not accurate, density-sensitive lines, and lines whose fluxes are incompatible with other lines of the same ion. With regard to the latter point, we recall that the inverse emissivity curves (i.e. the ratios between the line fluxes and emissivity functions) should be similar for all the lines of the same ion. We therefore excluded, for any given ion, those lines whose inverse emissivity curves differed from the others by more than a factor of $\sim 2$, suggesting likely problems either in the flux measurement or in the theoretical emissivity. Cases of only two lines for a given ion with discrepant curves did not occur.

The EMD reconstruction was carried out by applying to the measured line fluxes the Markov-Chain Monte Carlo (MCMC) method by Kashyap \& Drake (1998). This algorithm performs a random sampling of the EMD and abundances parameter space, with the aim of maximizing the probability of obtaining the best match between predicted and measured line fluxes. The MCMC method yields a volume emission measure distribution, $E M(T)$, sampled over a pre-defined temperature grid, and elemental abundances relative to iron, with the associated statistical uncertainties. The iron abundance is then estimated by comparing the observed spectrum with a set of simulated spectra computed from the derived EMD for different metallicities. The adopted temperature grid ranges from $\log T=6.4$ to $\log T=7.5$ with intervals $\Delta \log T=0.1$. The chosen limits in $\log T$ are dictated by the range of temperatures of maximum emissivity of the lines used for the EMD reconstruction $(\log T=6.5-7.3)$ and the typical width of the emissivity curves. Note also that the algorithm can assign uncertainties to the emission measure at a given temperature only if there is a sufficient number of lines that form around that temperature and can therefore provide information on the plasma emissivity.

The EMD and abundances derived in the first iteration have been used to compute a new continuum level, which resulted slightly different from that estimated from the MOS 3-T model. Therefore the procedure described above was repeated to derive a new solution. The EMD and abundances resulting from this second iteration describe sufficiently well the observed fluxes, within a factor of 2 , and give a continuum level consistent with that employed for the line measurement. See however Sect. 4 for a detailed discussion about the effect on the continuum level of high-temperature plasma not accessible by the RGS.

\section{EPIC light curve and spectrum of SU Aur}

Figure 2 shows the combined background-subtracted MOS1+MOS2 light curve of SU Aur. The star was highly variable during the observation, with three flares occurring at nearly equal intervals of $\sim 40 \mathrm{ks}$, and lasting $\sim 15-20 \mathrm{ks}$ each. The first flare was the strongest one, with a peak count rate a factor of $\sim 3$ higher than the quiescent level observed outside of flares. The flare started from an enhanced level, with the 
Table 1. Results of the joint MOS1 and MOS2 spectral fits of SU Aur. The intervals for time-dependent analysis are indicated in Fig. 2. Errors are computed with $\Delta \chi^{2}=1$, allowing all parameters to vary. When no error is given, the parameter was kept fixed to the tabulated value.

\begin{tabular}{|c|c|c|c|c|c|c|c|c|c|}
\hline & Total & $Q$ & $D 0$ & $R 1$ & $P 1$ & $D 1$ & $P 2$ & D2 & $P 3$ \\
\hline$N_{\mathrm{H}} \quad\left(10^{21} \mathrm{~cm}^{-2}\right)$ & $3.34_{-0.05}^{+0.11}$ & 3.34 & 3.34 & 3.34 & 3.34 & 3.34 & 3.34 & 3.34 & 3.34 \\
\hline$T_{1} \quad(\mathrm{keV})$ & $0.67_{-0.01}^{+0.01}$ & $0.66_{-0.01}^{+0.02}$ & $0.67_{-0.06}^{+0.04}$ & $0.68_{-0.04}^{+0.05}$ & $0.66_{-0.06}^{+0.08}$ & $0.61_{-0.11}^{+0.05}$ & $0.68_{-0.04}^{+0.04}$ & $0.81_{-0.03}^{+0.02}$ & $0.65_{-0.03}^{+0.02}$ \\
\hline$T_{2} \quad(\mathrm{keV})$ & $1.64_{-0.07}^{+0.01}$ & $1.54_{-0.16}^{+0.01}$ & $1.86_{-0.16}^{+0.28}$ & $1.90_{-0.18}^{+0.20}$ & $1.60_{-0.16}^{+0.20}$ & $1.62_{-0.42}^{+0.11}$ & $1.72_{-0.38}^{+0.04}$ & $1.73_{-0.43}^{+0.03}$ & $1.81_{-0.17}^{+0.20}$ \\
\hline$T_{3} \quad(\mathrm{keV})$ & $\begin{array}{r}-0.07 \\
4.99_{-0.59}^{+0.74}\end{array}$ & $3.84_{-0.82}^{+1.08}$ & $6.21_{-1.07}^{+2.16}$ & $10.84_{-3.11}^{+10.67}$ & $11.72^{+18.48}$ & $4.54_{-1.14}^{+2.33}$ & $4.32_{-1.02}^{+4.03}$ & $3.01^{+1.26}$ & $5.43^{+7.19}$ \\
\hline$E M_{1}\left(10^{53} \mathrm{~cm}^{-3}\right)$ & $1.34_{-0.12}^{+0.12}$ & $1.23_{-0.02}^{+0.05}$ & $1.15_{-0.14}^{+0.07}$ & $1.41_{-0.14}^{+0.19}$ & $1.58_{-021}^{+0.263}$ & $1.70_{-0.11}^{+0.21}$ & $1.37_{-0.14}^{+0.02}$ & $1.48_{-0.16}^{+0.213}$ & $1.55_{-0.09}^{+0.76}$ \\
\hline$E M_{2} \quad\left(10^{53} \mathrm{~cm}^{-3}\right)$ & $2.61_{-024}^{+0.21}$ & $1.81_{-0.45}^{+0.025}$ & $4.62_{-0.66}^{+0.14}$ & $5.21_{-0.52}^{+0.54}$ & $5.06_{-0.92}^{+1.21}$ & $3.65^{+2.26}$ & $2.83_{-179}^{+1.155}$ & $1.01_{-0.21}^{+2.64}$ & $3.06_{-0.94}^{+1.69}$ \\
\hline$E M_{3} \quad\left(10^{53} \mathrm{~cm}^{-3}\right)$ & $1.41_{-0.19}^{+0.24}$ & $0.94_{-0.33}^{+0.53}$ & $2.45_{-0.61}^{+0.56}$ & $1.96_{-0.49}^{+0.52}$ & $\begin{array}{l}-0.92 \\
4.48_{-0.64}^{+0.75}\end{array}$ & $\begin{array}{l}-2.21 \\
3.39_{-2.00}^{+2.50}\end{array}$ & $2.73_{-1.51}^{+1.79}$ & $2.61_{-1.01}^{+1.11}$ & $\begin{array}{l}-0.94 \\
1.48_{-0.71}^{+0.97}\end{array}$ \\
\hline $\mathrm{O}$ & $0.33_{-0.12}^{+0.19}$ & $0.33^{-0.33}$ & $0.33^{-0.61}$ & $0.33^{-0.49}$ & $0.33^{-0.64}$ & $0.33^{-2.00}$ & $0.33^{-1.51}$ & $0.33^{-1.01}$ & $0.33^{-0 . / 1}$ \\
\hline $\mathrm{Ne}$ & $1.29_{-0.24}^{+0.125}$ & 1.29 & 1.29 & 1.29 & 1.29 & 1.29 & 1.29 & 1.29 & 1.29 \\
\hline $\mathrm{Mg}$ & $1.14^{+0.13}$ & 1.14 & 1.14 & 1.14 & 1.14 & 1.14 & 1.14 & 1.14 & 1.14 \\
\hline $\mathrm{Si}$ & $0.59_{-0.06}^{+0.13}$ & 0.59 & 0.59 & 0.59 & 0.59 & 0.59 & 0.59 & 0.59 & 0.59 \\
\hline $\mathrm{S}$ & $0.40_{-0.07}^{+0.06}$ & 0.40 & 0.40 & 0.40 & 0.40 & 0.40 & 0.40 & 0.40 & 0.40 \\
\hline $\mathrm{Fe}$ & $0.58_{-0.05}^{+0.04}$ & 0.58 & 0.58 & 0.58 & 0.58 & 0.58 & 0.58 & 0.58 & 0.58 \\
\hline$\chi_{r}^{2}$ & $1.15^{-0.05}$ & 1.10 & 0.94 & 0.92 & 1.01 & 1.08 & 1.01 & 0.98 & 0.98 \\
\hline d.o.f. & 563 & 357 & 118 & 126 & 107 & 105 & 144 & 152 & 176 \\
\hline$F_{\mathrm{X}}^{a} \quad\left(10^{-12} \mathrm{erg} \mathrm{cm}^{-2} \mathrm{~s}^{-1}\right)$ & 3.4 & 2.6 & 5.1 & 5.4 & 7.3 & 5.6 & 4.4 & 3.2 & 3.9 \\
\hline$L_{\mathrm{X}}^{a}\left(10^{30} \mathrm{erg} \mathrm{s}^{-1}\right)$ & 8.1 & 6.1 & 12.0 & 12.7 & 17.2 & 13.2 & 10.4 & 7.5 & 9.2 \\
\hline
\end{tabular}

${ }^{a}$ Unabsorbed X-ray flux and luminosity in the $0.3-8 \mathrm{keV}$ band.

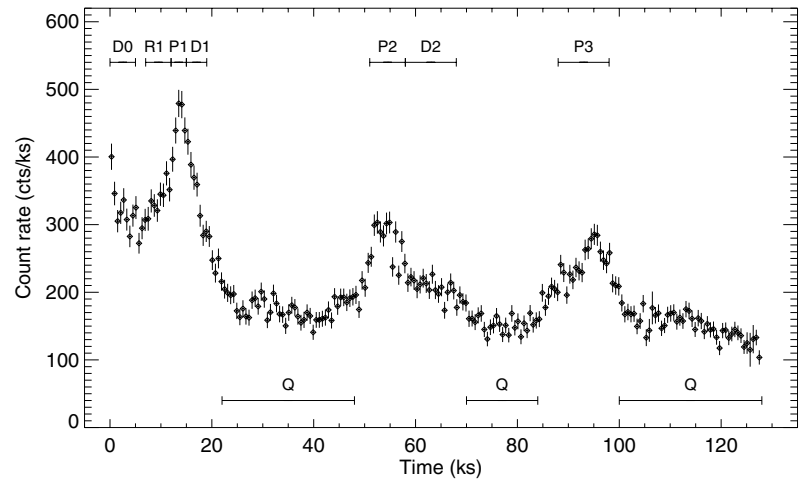

Fig. 2. Combined MOS1+MOS2 light curve of SU Aur, binned in $600 \mathrm{~s}$ intervals. The intervals where time-dependent spectral analysis has been performed are indicated by horizontal segments.

emission decreasing and reaching a minimum about $5 \mathrm{ks}$ after the beginning of the observation, probably representing the decay phase of a previous flare. The other two flares had similar amplitudes, with peak count rate a factor of $\sim 2$ higher than the quiescent level. Exponential fits of the rise and decay phases of the three flares give similar rise times of 5.5-6 ks for all flares, and decay times of 5.6, 9 and $5 \mathrm{ks}$, respectively.

In order to compare in a consistent way the MOS results with those obtained from the RGS analysis, we extracted the total MOS1 and MOS2 spectra integrated over the entire observation. The MOS spectra (Fig. 3) are quite hard and show a strong $\mathrm{Fe} 6.7 \mathrm{keV}$ line, clearly indicating the presence of hot plasma. The results of the spectral fit are given in Table 1 . The fit confirms the presence of hot plasma, with significant amounts of material up to $5 \mathrm{keV}(\sim 60 \mathrm{MK})$ and comparable emission measures of the three components $\left(E M_{2} / E M_{1} \sim 2\right.$ and $\left.E M_{3} / E M_{1} \sim 1\right)$. The best-fit column density is $N_{\mathrm{H}}=$ $3.3 \times 10^{21} \mathrm{~cm}^{-2}$ in agreement with previous X-ray observations (Wichmann et al. 1996; Skinner \& Walter 1998). Abundances are subsolar (0.3-0.6 times the solar photospheric values) with the exception of $\mathrm{Ne}$ and $\mathrm{Mg}$ that are consistent with the solar value. The total $\mathrm{X}$-ray luminosity in the $0.3-8 \mathrm{keV}$ band is $L_{\mathrm{X}} \sim 8 \times 10^{30} \mathrm{erg} \mathrm{s}^{-1}$. We note that our results are in excellent

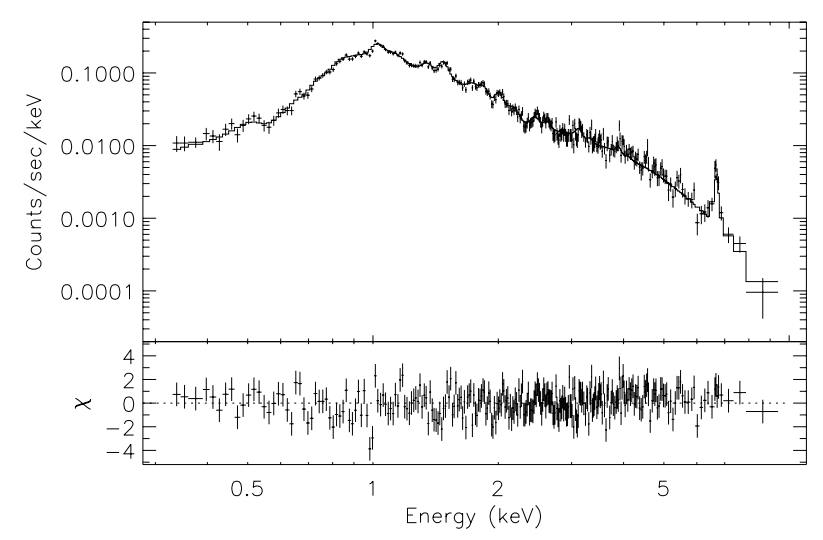

Fig. 3. MOS1 spectrum of SU Aur for the entire observation. The MOS2 spectrum is very similar and is not shown for clarity. The best-fit model from the joint MOS1+MOS2 fit is also shown. In the bottom panel the residuals in units of $\chi$ are plotted.

agreement with those obtained by Robrade \& Schmitt (2006) from their joint MOS and RGS fit.

\subsection{Time-dependent spectral analysis}

The high count rate of SU Aur allows us to perform a detailed time-resolved spectral analysis of the quiescent and flaring emission to study the variations with time of the coronal properties. We have extracted MOS 1 and MOS2 spectra for eight time intervals, indicated in Fig. 2: the presumably quiescent level observed after each flare (the three segments labeled "Q"), the decay observed before the first flare ("D0"), the rise, peak and decay of the first flare, ("R1", "P1" and "D1"), the peak and decay of the second flare ("P2" and "D2") and the peak of flare 3 ("P3"). For the spectral fits in each time interval, individual abundances were initially left free to vary. However, we found no significant differences, within the errors, with the abundances derived from the total spectrum. Similarly, no significant variations were found in the hydrogen column density. To better constrain the other parameters, we therefore fixed all abundances and $N_{\mathrm{H}}$ to the values 


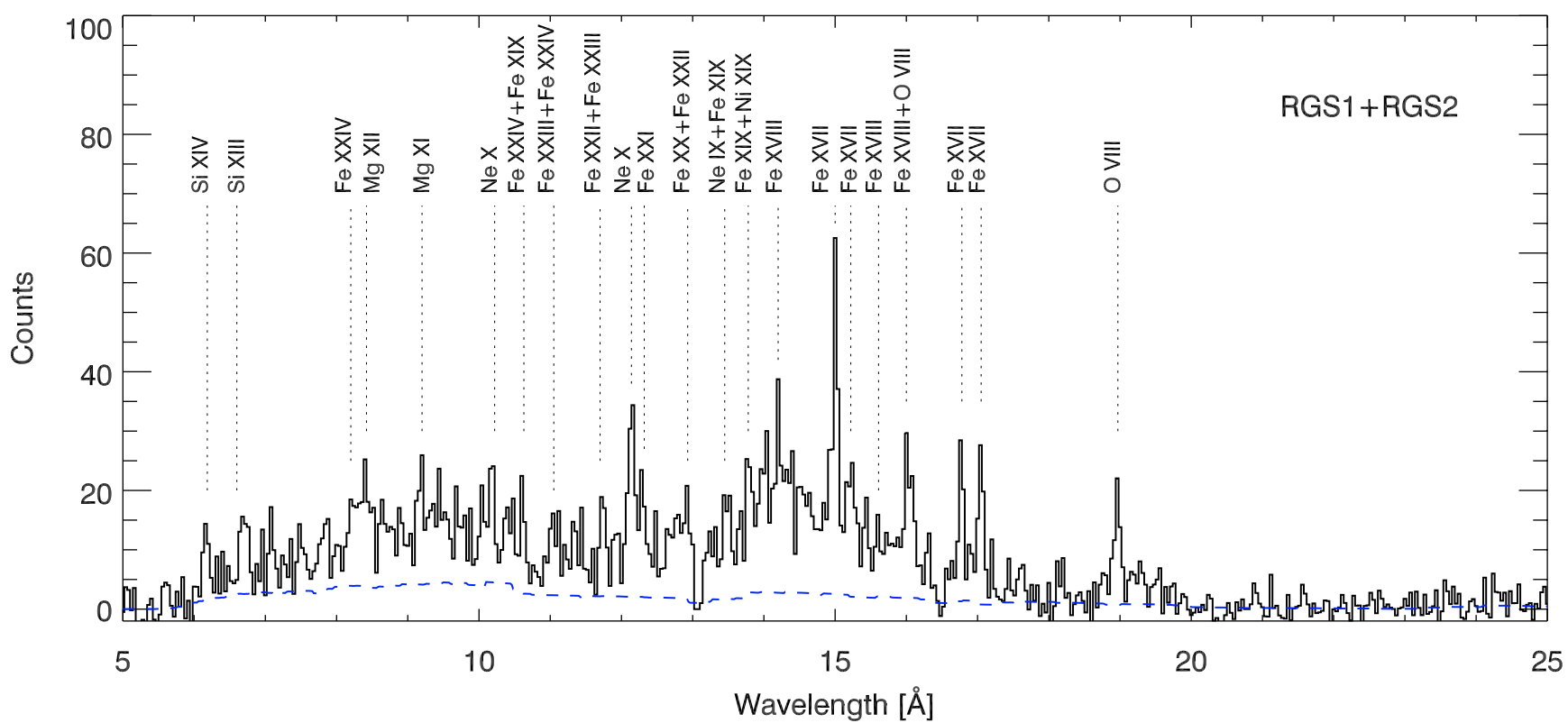

Fig. 4. Co-added RGS1 and RGS2 spectra of SU Aur. For a better display the summed spectrum has been rebinned with a bin size of $0.04 \AA$. The dashed line shows the adopted continuum level. The main lines identified in the spectrum are also indicated. Note that the apparent weakness of the spectrum between 10.5 and $13.5 \AA$ is due to the lack of RGS1 data in this wavelength range because of the failure of CCD 7 .

obtained from the total spectrum, and repeated the fits. The resulting best-fit parameters are given in Table 1.

The quiescent spectrum is characterized by plasma at temperatures of $0.7,1.5$ and $3.8 \mathrm{keV}(\sim 8,18$ and $45 \mathrm{MK})$, with comparable amounts of material at all temperatures $\left(E M_{2} / E M_{1} \sim 1.5\right.$ and $\left.E M_{3} / E M_{1} \sim 0.8\right)$, and an X-ray luminosity of $6 \times 10^{30} \mathrm{erg} \mathrm{s}^{-1}$. The temperature and emission measure of the coolest component remain nearly constant during the whole observation, with variations by at most $20-40 \%$. On the other hand, significant increases of the hottest temperature and of the emission measures of the two hotter components are observed during the flares. During the initial decay the plasma has $T_{3} \sim 70 \mathrm{MK}$, and emission measures a factor of 2.5 higher than the quiescent level. The first flare is very hot, reaching a maximum temperature of $\sim 135 \mathrm{MK}$ at the flare peak, where also the emission measure peaks, with $E M_{2} \sim E M_{3} \sim 5 \times 10^{53} \mathrm{~cm}^{-3}$, a factor of 3-5 higher than in quiescence. The peak luminosity of the flare is $L_{\mathrm{X}} \sim 2 \times 10^{31} \mathrm{erg} \mathrm{s}^{-1}$. The temperature then returns rapidly to the quiescent level in the decay phase, while the emission measure is still high. The other two weaker flares, on the contrary, do not show strong variations of the plasma temperature, which is $\sim 50-60 \mathrm{MK}$ at the peak, not much higher than the quiescent value, and their evolution is mainly due to the variations of the emission measure. Both flares have similar peak luminosities $L_{\mathrm{X}} \sim 1 \times 10^{31} \mathrm{erg} \mathrm{s}^{-1}$.

\section{The RGS spectrum of SU Aur}

Figure 4 shows the co-added RGS1 and RGS2 spectra of SU Aur. The RGS spectrum of SU Aur shows a strong continuum below $\sim 15 \AA$ and weak lines, mostly from Fe XVII-XXIV; other prominent lines are H-like and He-like lines of Si XIV-XIII, Mg XII-XI, NeX-IX, and O VIII. Above $20 \AA$ the emission is strongly suppressed, with no lines visible above the noise level. All the observed spectral characteristics can be attributed to the presence of hot plasma, to the absence of large amounts of cool plasma, and to the high column density, as shown below. In fact, if we use the plasma parameters derived from the best-fit of

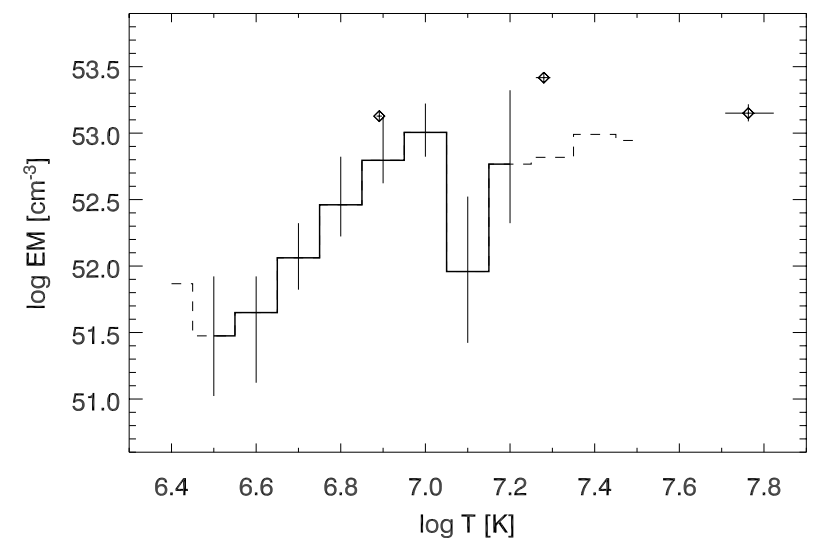

Fig. 5. Emission measure distribution of SU Aur derived from the RGS analysis. Unconstrained EMD components are indicated with a dashed line. The results of the 3-temperature MOS fit are also plotted for comparison (diamonds).

the MOS spectra to simulate the RGS spectrum, we find a very good agreement between the predicted RGS spectrum and the observed one. Since the O VII He-like triplet is not detected and the other He-like triplets in the RGS spectra are too weak and unresolved (Si XIII and Mg XI) or severely blended with Fe lines (Ne IX), we have not the possibility of measuring the plasma density in SU Aur.

Table 2 lists the strongest lines (with $S / N>1.5$ ) identified in the RGS spectrum, with their fluxes measured in the second iteration of the EMD reconstruction process; lines selected for the EMD reconstruction are marked by an asterisk. The final EMD is shown in Fig. 5, where we also plot for comparison the results of the MOS 3-T fit. Error bars represent the central $68 \%$ of the distribution of values randomically sampled by the MCMC method. The algorithm cannot assign uncertainties to the EM values outside the range $6.5 \leq \log T \leq 7.2$, because of the paucity of information from the RGS spectrum at these temperatures. Figure 6 


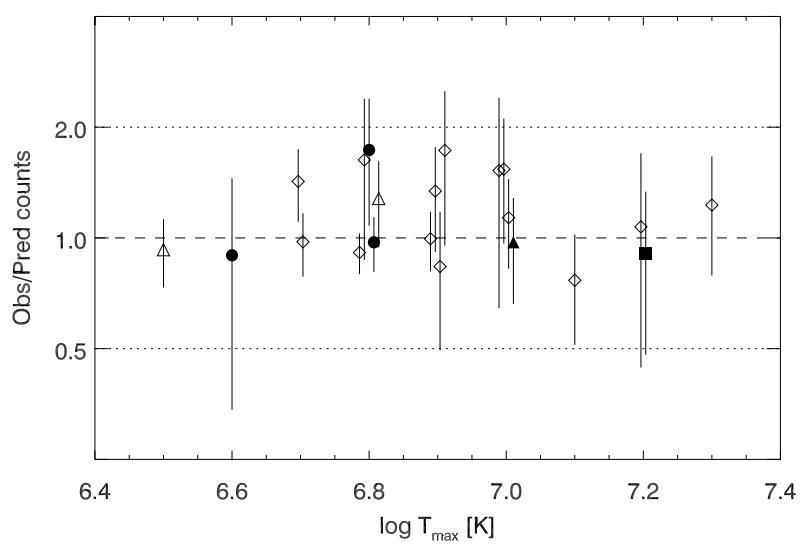

Fig. 6. Comparison between the observed and predicted fluxes for the lines used in the EMD reconstruction. Points relative to lines formed at the same $\log T_{\max }$ have been slightly displaced for clarity. Fe: open diamonds; Ne: filled circles; $\mathrm{O}$ : open triangles; $\mathrm{Mg}$ : filled triangle; $\mathrm{Si}:$ filled square.

shows the ratio of observed to predicted line fluxes for the lines used in the EMD reconstruction.

The EMD has a maximum at $\log T=7.0$ and, below the peak, decreases with a trend $\propto T^{\alpha}$, with $\alpha=3.2 \pm 0.7$. There is an indication of significant emission measure, at a level comparable to the peak, up to $\log T=7.5$. The presence of such a hot tail appears to be reliable on the basis of the results of the MOS fits (Fig. 5). Moreover, the third component of the MOS model indicates the presence of very hot plasma at $\log T \sim 7.8$. The emission measure of this component cannot be investigated with the RGS, because of the paucity of hot lines in the RGS spectrum and of the limited spectral band, that does not allow us to detect the continuum level at high energies $(E \gtrsim 2 \mathrm{keV})$. Our EMD solution indeed underestimates the MOS spectra above $\sim 3.5 \mathrm{keV}$. This discrepancy can be solved by adding to the derived EMD an isothermal component with temperature equal to that of the third MOS component and emission measure adjusted to fit the MOS spectra. Adding a hotter component to the EMD results in an increase of both the predicted continuum and of the predicted line fluxes for lines formed at high temperatures. In our case, we have verified that both effects are small and negligible above $\sim 11 \AA$. At shorter wavelengths, for the lines used in the EMD reconstruction and abundance estimates, we find that the only line significantly affected is the Si XIV $6.19 \AA$ line, whose measured flux is reduced by $\sim 10 \%$ as a consequence of the stronger continuum, and the predicted flux is increased by $\sim 15 \%$. Since this is the only line used for $\mathrm{Si}$, the global effect is only to reduce the Si abundance by $\sim 30 \%$ without affecting the shape of the EMD ${ }^{1}$. We also note that the constrained part of the EMD is not significantly affected by the flares, and is therefore likely to represent the emission from quiescent structures in the corona of SU Aur. In fact, as shown from the time-dependent analysis of the MOS spectra, the cool component at $\log T \sim 6.9$ does not change during the flares, with the flaring emission only due to the variations of the hotter components, at temperatures well above the EMD peak where the RGS is not sensitive.

The strong absorption prevents the detection of the O VII lines that, together with the slightly hotter O VIII lines,

\footnotetext{
1 Note that when, for a given element, only lines forming at the same temperature are used in the EMD reconstruction, these lines are employed by the MCMC algorithm essentially to derive the abundance of that element, and not to add new information on the EMD.
}

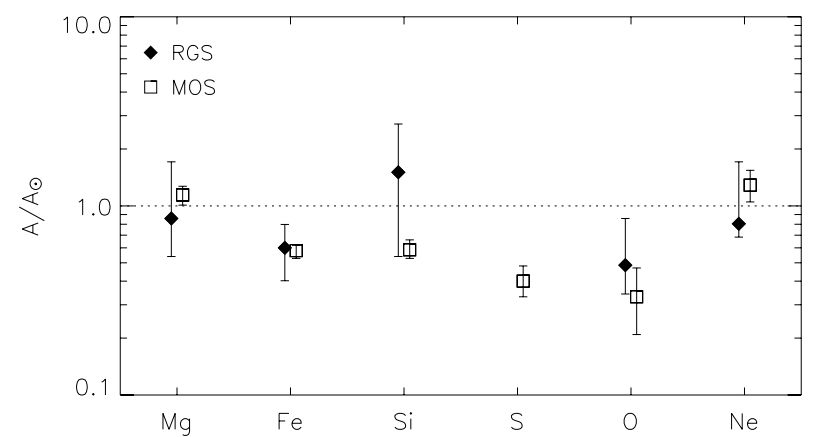

Fig. 7. Elemental abundances of SU Aur, relative to the solar abundances of Anders \& Grevesse (1989), plotted in order of increasing FIP. Filled diamonds and open squares refer to the abundances derived from RGS and MOS, respectively.

would have allowed us to extend the EMD to temperatures lower than $\sim 3 \mathrm{MK}$. Nevertheless, we can estimate a rough upper limit to the amount of cool plasma at $\log T=6.3$ by means of the MOS spectra, where the low-energy tail has a significant signal in spite of the relatively high value of $N_{\mathrm{H}}$ (Fig. 3). The EMD shown in Fig. 5 describes sufficiently well the MOS spectra. The addition of an isothermal component at $\log T=6.3$ results in a contribution to the X-ray emission essentially at low energies $(E \lesssim 0.8 \mathrm{keV}$ ), which is however strongly suppressed by absorption. Using therefore the spectral range $E<0.8 \mathrm{keV}$, we find that this contribution can be discerned in the MOS spectra, at the $3 \sigma$ level, when its emission measure is at least $\sim 2.5 \times 10^{52} \mathrm{~cm}^{-3}$. The unabsorbed luminosity arising from such an amount of plasma at $\log T=6.3$, in the $0.3-8 \mathrm{keV}$ band, would be $\sim 2.3 \times 10^{29} \mathrm{erg} \mathrm{s}^{-1}$, i.e. only $\sim 3 \%$ of the luminosity of the total EMD shown in Fig. 5 $\left(L_{\mathrm{X}} \sim 8.4 \times 10^{30} \mathrm{erg} \mathrm{s}^{-1}\right)$. Even if we use for $N_{\mathrm{H}}$ the upper value of the confidence range given in Table 1, we obtain an upper limit to the emission measure of this component of $6 \times 10^{52} \mathrm{~cm}^{-3}$, corresponding to $5 \%$ of the total luminosity. This upper limit at $\log T=6.3$ is also still consistent with the observed RGS spectrum: the addition of this cool component to the EMD does not produce significant changes of the predicted RGS spectrum.

The abundances, relative to iron and to the solar values, derived from the EMD reconstruction are: $\mathrm{O} / \mathrm{Fe}=0.81$ $[0.57-1.43], \quad \mathrm{Ne} / \mathrm{Fe}=1.38 \quad[1.14-2.85], \quad \mathrm{Mg} / \mathrm{Fe}=1.43$ [0.90-2.85], $\mathrm{Si} / \mathrm{Fe}=2.51 \quad[0.90-4.52]$, where ranges in parenthesis are $68 \%$ confidence intervals. The iron abundance is estimated to be $0.6 \pm 0.2$. Figure 7 shows the abundances derived from the RGS analysis, compared with those derived from MOS, plotted in order of increasing First Ionization Potential (FIP). Note that $\mathrm{S}$ is not detected in the RGS spectrum and was determined from MOS only. We find a good agreement, within the errors, between the RGS and MOS abundances. The abundance of $\mathrm{Si}$ is poorly determined by the RGS, and its value is too high, although still compatible with the MOS one, given the large error. This is due to the fact that the Si XIV line used to determine the $\mathrm{Si}$ abundance forms at high temperatures, where the RGS is not able to fully constrain the EMD, and to the poor effective area calibration of the RGS below $7 \AA$. As explained above, the addition of a hotter component to the EMD in order to obtain a good fit of the MOS data at the highest energies results in a slightly lower $\mathrm{Si}$ abundance ( $\sim$ solar $)$, in better agreement with the MOS Si abundance.

Telleschi et al. (2007b) analysed the same XMM-Newton data with a simultaneous fitting of the RGS and MOS spectra assuming a triangular EMD consisting of two power-laws with fixed ascending slope $\left(\propto T^{2}\right)$. They found similar results in terms 
Table 3. X-ray properties of known Taurus-Auriga members and of other stars detected in our observation. For the last three members we give the position of the nearest X-ray source, even if its distance is greater than $4^{\prime \prime}$.

\begin{tabular}{|c|c|c|c|c|c|c|c|c|}
\hline Name & Sp.T. & $\begin{array}{l}\mathrm{RA}_{\mathrm{X}} \\
(2000)\end{array}$ & $\begin{array}{l}\operatorname{Dec}_{X} \\
(2000)\end{array}$ & $\begin{array}{c}\Delta r^{a} \\
\left({ }^{\prime \prime}\right)\end{array}$ & $\begin{array}{l}\text { Offset }^{b} \\
\left({ }^{\prime \prime}\right)\end{array}$ & Sign. ${ }^{c}$ & $\begin{array}{r}\text { Count rate } \\
(\mathrm{cts} / \mathrm{ks})\end{array}$ & Notes \\
\hline \multicolumn{9}{|c|}{ known Taurus-Auriga members } \\
\hline AB Aur & B9 & 45545.83 & +303304.2 & 0.97 & 0.13 & 90.7 & $12.65 \pm 0.36$ & $\mathrm{HAeBe}$ \\
\hline SU Aur & G2III & 45559.32 & +303401.6 & 0.71 & 0.82 & 438.1 & $232.57 \pm 1.44$ & CTTS \\
\hline HBC 427 & $\mathrm{~K} 7$ & 45601.97 & +302103.8 & 0.87 & 0.57 & 266.9 & $269.14 \pm 2.71$ & WTTS \\
\hline 2MASS J04554801+3028050 & M5.6 & 45547.77 & +302803.1 & 3.00 & 3.68 & 29.2 & $3.96 \pm 0.27$ & \\
\hline 2MASS J04554757+3028077 & M4.75 & & & & 5.29 & & & \\
\hline 2MASS J04552333+3027366 & M6.25 & 45522.98 & +302739.7 & 4.08 & 5.53 & 6.5 & $0.76 \pm 0.18$ & \\
\hline 2MASS J04554046+3039057 & M5.25 & 45540.27 & +30 3909.9 & 3.52 & 4.86 & 5.7 & $0.45 \pm 0.15$ & $d$ \\
\hline \multicolumn{9}{|c|}{ other identifications } \\
\hline JH 431 & & 45522.71 & +303807.9 & 2.45 & 0.97 & 44.0 & $8.64 \pm 0.40$ & \\
\hline JH 428 & & 45531.77 & +303001.0 & 2.12 & 0.67 & 29.1 & $3.51 \pm 0.24$ & \\
\hline JH 433 & & 45556.02 & +303621.3 & 1.97 & 0.61 & 36.7 & $3.02 \pm 0.17$ & $e$ \\
\hline HD 31305 & A0 & 45548.18 & +302016.0 & 1.12 & 0.86 & 136.3 & $90.26 \pm 1.68$ & IR excess \\
\hline RXJ $0456.5+3023$ & M3 & 45632.05 & +302325.3 & 2.83 & 1.12 & 32.1 & $15.02 \pm 0.90$ & no $\mathrm{Li}$ \\
\hline GY Aur & A8+G5IV & 45648.56 & +303536.4 & 2.06 & 0.33 & 33.1 & $5.17 \pm 0.33$ & Algol-type \\
\hline 2MASS J04551407+3027272 & & 45514.03 & +302727.8 & 2.47 & 0.79 & 11.6 & $1.27 \pm 0.23$ & \\
\hline 2MASS J04553249+3040495 & & 45532.57 & +304047.7 & 3.91 & 2.08 & 6.2 & $0.51 \pm 0.11$ & \\
\hline 2MASS J04554820+3030160 & & 45548.15 & +303015.7 & 2.37 & 0.82 & 22.9 & $1.72 \pm 0.15$ & $e$ \\
\hline 2MASS J04560534+3025393 & & 45605.21 & +302539.2 & 4.34 & 1.78 & 5.3 & $0.55 \pm 0.14$ & \\
\hline 2MASS J04560916+3045179 & & 45609.08 & +304517.7 & 4.42 & 1.14 & 10.7 & $1.39 \pm 0.20$ & \\
\hline 2MASS J04560960+3029318 & & 45609.64 & +302927.9 & 2.30 & 3.98 & 11.2 & $0.56 \pm 0.09$ & \\
\hline 2MASS J04561006+3031235 & & 45610.17 & +303123.8 & 3.76 & 1.32 & 7.3 & $0.35 \pm 0.07$ & \\
\hline 2MASS J04561535+3022268 & & 45615.49 & +302226.3 & 3.24 & 1.84 & 9.9 & $1.32 \pm 0.20$ & \\
\hline 2MASS J04561686+3026528 & & 45617.06 & +302653.8 & 3.61 & 2.72 & 17.8 & $2.12 \pm 0.20$ & \\
\hline 2MASS J04561986+3030047 & & 45620.06 & +303005.7 & 1.84 & 2.66 & 19.7 & $1.26 \pm 0.13$ & \\
\hline 2MASS J04562475+3034576 & & 45624.95 & +303457.6 & 2.78 & 2.56 & 16.6 & $1.34 \pm 0.15$ & \\
\hline 2MASS J04562751+3033420 & & 45627.51 & +303340.7 & 1.66 & 1.38 & 6.5 & $0.16 \pm 0.05$ & \\
\hline 2MASS J04564128+3041347 & & 45641.31 & +304135.7 & 2.15 & 1.04 & 5.7 & $0.30 \pm 0.08$ & \\
\hline 2MASS J04564132+3024575 & & 45641.44 & +302456.1 & 2.09 & 2.19 & 30.2 & $5.66 \pm 0.38$ & \\
\hline 2MASS J04565038+3028091 & & 45650.29 & +302810.7 & 2.87 & 1.91 & 6.3 & $0.51 \pm 0.12$ & \\
\hline 2MASS J04565237+3038186 & & 45652.39 & +303817.2 & 2.93 & 1.49 & 6.4 & $0.44 \pm 0.11$ & \\
\hline
\end{tabular}

${ }^{a}$ X-ray position error; ${ }^{b}$ offset between the X-ray and optical position; ${ }^{c}$ significance of detection; ${ }^{d}$ source detected on MOS 1 only; ${ }^{e}$ candidate $^{-}$ Taurus-Auriga member according to Scelsi et al. (2007).

of abundances and peak temperature, although their EMD peaks at a slightly lower temperature $(\log T \sim 6.9)$ and the $\mathrm{Ne}$ abundance (0.38 solar, in units of Anders \& Grevesse 1989) is significantly lower than ours. This discrepancy could be abscribed to the different EMD shape assumed by Telleschi et al. (2007b), which has more emitting material in the range of temperatures where $\mathrm{Ne}$ lines form, thus reducing the required $\mathrm{Ne}$ abundance, and/or to the different approach used.

\section{Other sources in the field of view}

The list of $104 \mathrm{X}$-ray sources derived from the source detection process (Sect. 2.1) has been cross-correlated with the list of known Taurus-Auriga members and other objects in the field, and with the 2MASS catalogue, to search for optical/IR counterparts. We adopted a search radius of $4^{\prime \prime}$, derived from the cumulative distribution function of the offsets between the X-ray and optical positions, following Randich \& Schmitt (1995). With this choice we expect to find most true identifications, and the expected number of spurious matches is relatively low ( 4). Using this search radius, we identify 4 sources with known TaurusAuriga PMS stars (see Table 3). Three other low-mass TaurusAuriga members fall in the EPIC field of view: for them, we find an X-ray source at a slightly larger distance $\left(\sim 5-5.5^{\prime \prime}\right)$, one of which already identified with another member. Examining the EPIC image, we see that the latter three sources fall very close to CCD gaps in one or both MOS instruments, so that their derived X-ray position might not be accurate. We therefore decided to retain them as possible identifications. All these sources are also reported by Güdel et al. (2007a) as part of the XMM-Newton Extended Survey of the Taurus molecular cloud (XEST). Additional 6 sources are identified with other known stars in the field, likely not associated with the Taurus-Auriga star-forming region or with no indication of membership, while for other 16 sources we find a counterpart in the 2MASS catalogue. In total, we identified $28 \mathrm{X}$-ray sources, listed in Table 3. The count rate reported in the table is derived from the merged dataset, except one case where the source was detected on MOS1 only, and is expressed as equivalent count rate for a single MOS instrument.

\subsection{Bright sources}

Apart from SU Aur, three of the detected sources are sufficiently bright to allow reliable spectral analysis, i.e. the Taurus-Auriga members HBC 427 and AB Aur, and the star HD 31305. A detailed spectral analysis of the two members has already been performed in the framework of the XEST survey by Franciosini et al. (2007) and Telleschi et al. (2007c), and therefore will not be repeated here; we summarize their results below. 


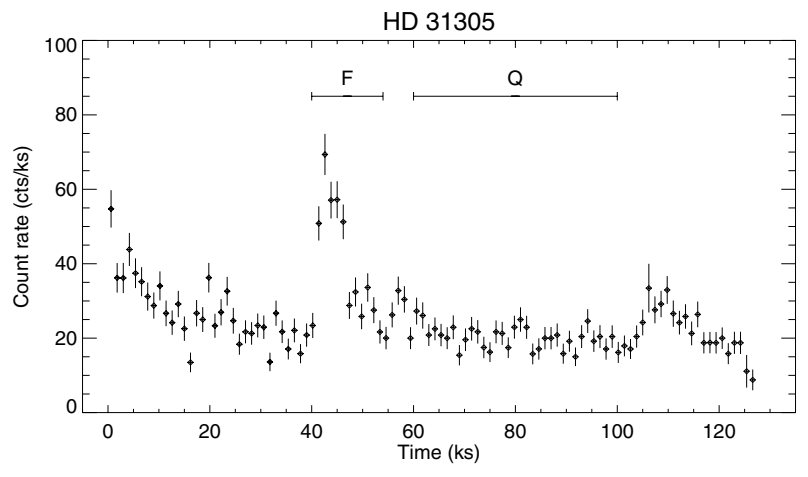

Fig. 8. MOS1+MOS2 light curve of HD 31305. The intervals used for time-dependent spectral analysis are also shown.

The WTT star HBC 427 displayed a strong long-lasting flare during the observation (see Fig. 2 in Franciosini et al. 2007), with the count rate increasing by a factor of $\sim 5$ in $\sim 2 \mathrm{~h}$, and returning to the quiescent level nearly $16 \mathrm{~h}$ later. Franciosini et al. (2007) performed a detailed time-resolved spectral analysis of this source. For the quiescent corona they found temperatures of 8 and $25 \mathrm{MK}$ with nearly equal emission measures and an X-ray luminosity of $\sim 3.5 \times 10^{30} \mathrm{erg} \mathrm{s}^{-1}$. During the flare the temperature increased up to $70 \mathrm{MK}$, with a peak luminosity of $\sim 1.5 \times 10^{31} \mathrm{erg} \mathrm{s}^{-1}$. From the analysis of the flare decay, the above authors derived a size of about 2 stellar radii for the flaring structures.

The Herbig Ae star AB Aur showed a nearly constant emission level during the observation, with only a slow modulation possibly due to an uneven distribution of active regions on the stellar surface. Telleschi et al. (2007c) analyzed the EPIC and RGS spectra of $A B$ Aur, finding temperatures of 2-7 MK, significantly lower than those observed in SU Aur and in other latetype PMS stars, and an X-ray luminosity $L_{\mathrm{X}} \sim 4 \times 10^{29} \mathrm{erg} \mathrm{s}^{-1}$. These authors discussed several possible explanations for the origin of the X-ray emission of $\mathrm{AB}$ Aur, suggesting that the most plausible ones are either a solar-like corona or a magnetically confined wind.

HD 31305 is an A0 star showing an IR excess in the IRAS bands, indicative of the presence of circumstellar material at a temperature of $\sim 400 \mathrm{~K}$ (Oudmaijer et al. 1992), although it is not included in the list of known Taurus members. HD 31305 has been previously detected in X-rays by $A S C A$, with a $0.5-10 \mathrm{keV}$ count rate of $\sim 15 \mathrm{cts} \mathrm{ks}^{-1}$ (Skinner \& Walter 1998). The light curve (Fig. 8) shows three flares. The first one started before the beginning of the observation, and only the decay is observed. The second one, occurring at $t \sim 40 \mathrm{ks}$, increased the count rate by a factor of $\sim 4$ in $\lesssim 1 \mathrm{~h}$, followed by a decay of $\sim 5 \mathrm{~h}$. A weaker flare is observed at the end of the observation. We performed spectral analysis for the second flare and the quiescent emission immediately after it. The best fit parameters are given in Table 4 . The quiescent temperatures are similar to those usually found in young late-type stars, with $T_{1}=1.0$ and $T_{2}=2.3 \mathrm{keV}$ ( 10 and $27 \mathrm{MK}$ ), and comparable emission measures. The flare is extremely hot, reaching a temperature of $8.6 \mathrm{keV}(100 \mathrm{MK})$, also typical of young late-type stars. The metallicity is strongly subsolar, with $Z=0.1 Z_{\odot}$, consistent with the values found in active late-type stars. The observed spectral characteristics suggest that the quiescent and flaring emission might be due to an unseen late-type companion, rather than to the A0 star itself.
Table 4. Best-fit parameters for the quiescent and flaring spectra of HD 31305. Errors are as in Table 1.

\begin{tabular}{lll}
\hline \hline & Quiescent & Flare \\
\hline$N_{\mathrm{H}}\left(10^{21} \mathrm{~cm}^{-2}\right)$ & $0.61_{-0.20}^{+0.34}$ & $0.63_{-0.35}^{+0.27}$ \\
$T_{1}(\mathrm{keV})$ & $1.04_{-0.19}^{+0.22}$ & $1.06_{-0.11}^{+0.17}$ \\
$T_{2}(\mathrm{keV})$ & $2.33_{-0.43}^{+2.34}$ & $8.62_{-3.53}^{+12.39}$ \\
$E M_{1}^{a}\left(10^{53} \mathrm{~cm}^{-3}\right)$ & $0.75_{-0.50}^{+1.15}$ & $3.13_{-2.05}^{+1.23}$ \\
$E M_{2}^{a}\left(10^{53} \mathrm{~cm}^{-3}\right)$ & $1.04_{-0.78}^{+0.38}$ & $1.06_{-0.28}^{+0.97}$ \\
$Z / Z_{\odot}$ & $0.12_{-0.07}^{+0.12}$ & $0.06_{-0.03}^{+0.11}$ \\
$\chi_{\mathrm{r}}^{2}$ & $1.40^{2}$ & 0.98 \\
d.o.f. & 65 & 44 \\
$F_{\mathrm{X}}^{b}\left(10^{-12} \mathrm{erg} \mathrm{cm}^{-2} \mathrm{~s}^{-1}\right)$ & 0.7 & 1.6 \\
$L_{\mathrm{X}}^{a, b}\left(10^{30} \mathrm{erg} \mathrm{s}^{-1}\right)$ & 1.6 & 3.6 \\
\hline
\end{tabular}

${ }^{a}$ Emission measures and luminosities are only indicative, being computed for the Taurus-Auriga distance, which might not be right for this star; ${ }^{b}$ unabsorbed flux and luminosity in the $0.3-8 \mathrm{keV}$ band.

\subsection{Other identified sources}

The other sources with an optical/infrared identification have less than $\sim 500$ cts in each MOS instrument, therefore we did not attempt a spectral analysis. For the three sources identified with the known low-mass members, spectral fits are reported by Güdel et al. (2007a) as part of XEST, who find temperatures of $\sim 10 \mathrm{MK}$ and X-ray luminosities in the range $1-6 \times 10^{28} \mathrm{erg} \mathrm{s}^{-1}$.

Of the other identified sources, only two, namely JH 433 and 2MASS J04554820+3030160, are possible candidate members of the Taurus-Auriga region, on the basis of their infrared photometry (Scelsi et al. 2007). JH 433 has also a measured proper motion which appears to be consistent with membership (Jones \& Herbig 1979). JH 428 and JH 431 have measured proper motions but no available photometric or spectroscopic data (Jones \& Herbig 1979), therefore no definitive conclusion on their membership status can be drawn. RXJ $0456.5+3023$ has $\mathrm{H} \alpha$ emission but no measurable Li line, and might be an older active star (Wichmann et al. 1996), while GY Aur is a candidate Algol-type binary (Budding et al. 2004). The remaining 2MASS objects have photometry inconsistent with membership and are likely to be older active field stars (Scelsi et al. 2007).

\subsection{Unidentified sources}

For the remaining 76 sources we found no known counterpart in any astronomical catalogue. Most of these sources are very faint, preventing a precise assessment of their nature. Although we cannot exclude that some of them might be yet unknown PMS objects, it is likely that most of them are background extragalactic objects. The sensitivity of our observation ranges from $0.3 \mathrm{cts} \mathrm{ks}^{-1}$ in the centre of the field to $0.7 \mathrm{cts} \mathrm{ks}^{-1}$ in the outer regions. Assuming a power-law spectrum with $\Gamma=1.4$ (see, e.g., Tozzi et al. 2001; Alexander et al. 2003) and the Galactic absorption towards SU Aur of $3 \times 10^{21} \mathrm{~cm}^{-2}$, we obtain a limiting flux of $\sim 0.5-1 \times 10^{-14} \mathrm{erg} \mathrm{cm}^{-2} \mathrm{~s}^{-1}$ in the $0.5-8 \mathrm{keV}$ band. Using the study of the extragalactic X-ray population in the $0.5-8 \mathrm{keV}$ band by Alexander et al. (2003), we estimate $\sim 80$ extragalactic $\mathrm{X}$-ray sources in our field, in very good agreement with the number of unidentified sources. This conclusion is also supported by Scelsi et al. (2007), who found that the bulk of sources detected in the XEST survey without 2MASS counterparts have energy distributions compatible with power-law spectra, characteristic of extragalactic objects, rather than with stellar thermal spectra. 


\section{Discussion and conclusions}

In this paper we have analyzed an XMM-Newton observation of the CTTS SU Aur and of the surrounding field, belonging to the Taurus-Auriga star-forming region. The thermal structure of the X-ray emitting plasma of the central star SU Aur was investigated using both EPIC low-resolution spectra and high-resolution RGS spectra. In particular, we derived for the first time the EMD of the star from the RGS spectra using a line-based method, and we performed a detailed time-dependent analysis of the EPIC spectra to study the variations of the plasma parameters in quiescence and during the flares. Spatial analysis of the EPIC data resulted in the detection of 104 X-ray sources, 6 of which are associated with the known Taurus-Auriga members in the field of view, including SU Aur.

The analysis of the XMM-Newton data presented here shows that very hot plasma is responsible for the observed X-ray emission of SU Aur. From the EPIC spectra we derived significant emission measure at temperatures from $\sim 8 \mathrm{MK}$ up to $\sim 40 \mathrm{MK}$ even in quiescence, consistently with the previous $A S C A$ and Chandra observations of SU Aur (Skinner \& Walter 1998; Smith et al. 2005), as well as with the analysis of the same XMM-Newton data performed by Robrade \& Schmitt (2006) and Telleschi et al. (2007b). The RGS-derived EMD, which describes quite well both EPIC and RGS spectra, shows that the bulk of the emission measure resides around $10 \mathrm{MK}$ and more, in agreement with the EPIC results. Such high temperatures found for SU Aur clearly indicate that the observed X-ray emission is mostly, if not entirely, of coronal origin, because shocks due to the infall of circumstellar material can heat the chromospheric plasma at temperatures of at most a few million degrees, for typical stellar parameters. An active corona is also witnessed by the presence of several flares during a $1.5 \mathrm{~d}$ observation.

Unfortunately, the high photoelectric absorption that strongly suppressed the RGS spectrum longward of $\sim 20 \AA$ does not allow us to check whether a contribution from an accretion shock is present also in this star. We note that a contribution from low-temperature plasma cannot be excluded from the fitting of the EPIC spectra. However, we estimated a rough upper limit to the emission measure of a cool component (at $\log T=6.3$ ) as a few times $10^{52} \mathrm{~cm}^{-3}$. Such a component, which might be of coronal and/or accretion origin, would contribute to the total $\mathrm{X}$-ray luminosity by less than $5 \%$, hence we conclude that any contribution from shock-heated plasma is not dominant in this star.

We now compare the EMD derived here for the corona of SU Aur with that obtained by Scelsi et al. (2005) for the WTTS HD 283572, also belonging to the Taurus-Auriga region (Fig. 9). The two distributions are very similar, both peaking at $\log T=7.0$, with significant high-temperature plasma, and showing a power-law increase between $\log T=6.5$ and 7.0. Although the best-fit slope for SU Aur is lower than that of HD 283572 ( $\sim 3$ for SU Aur, $\sim 5$ for HD 283572), the two EMDs are compatible within the errors. Similar emission measure distributions have been also found for other high-luminosity PMS and late-type stars (e.g. Sanz-Forcada et al. 2003; Argiroffi et al. 2003, 2005; Scelsi et al. 2005). This result suggests the presence of similar coronae in very active stars, and suggests also that the presence of the circumstellar disk does not influence significantly the properties of the coronal structures of SU Aur. Whether this is a common result should be investigated in the future by studying a larger sample of CTTS. We note that SU Aur is the only G-type CTTS studied so far at high-resolution, all

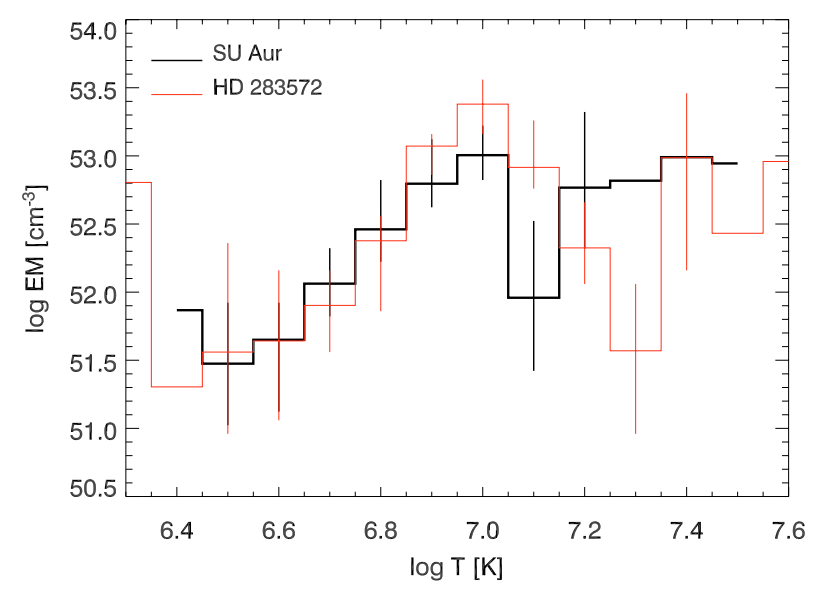

Fig. 9. Comparison of the EMD derived for SU Aur (black thick line) with that of the WTTS HD 283572 (red thin line) derived by Scelsi et al. (2005).

the other being K-type stars, therefore it is possible that it might have different coronal characteristics than later-type CTTS.

The shape of the EMD can give hints on the characteristics of the loop structures involved in the X-ray emission. In the case of a single hydrostatic loop with size lower than the pressure scale height, its EMD depends only on the maximum temperature $T_{\max }$, reached at the loop apex (Maggio \& Peres 1996), and can be approximated by a power-law $\propto T^{\alpha}$ for $T<T_{\max }$. Since the plasma is optically thin, the EMD of the whole stellar corona is the sum of the EMDs of the individual X-ray emitting loops; as shown by Peres et al. (2001), the total EMD resulting from an ensemble of loops with different $T_{\max }$ is still proportional to $T^{\alpha}$ for $T<\min \left\{T_{\max }\right\}$, while the shape at higher temperatures depends on the distribution in $T_{\max }$ of the loops. In the case of SU Aur, at temperatures of $\sim 10^{7} \mathrm{~K}$ the pressure scale height is $H_{\mathrm{p}} \simeq 5 \times 10^{3} T g_{\odot} / g_{\star} \sim 2 \times 10^{11} \mathrm{~cm}$, comparable to the stellar radius $\left(\log g_{\star}=3.6, R_{\star}=2.6 R_{\odot}\right.$, DeWarf et al. 2003). From the flare decay times and peak temperatures derived from the time-dependent analysis, using the relation by Serio et al. (1991) for a freely-decaying loop we can estimate upper limits to the flaring loop lenghts of $L \lesssim R_{\star}$. It is therefore reasonable to assume that the structures responsible for the observed X-ray emission of SU Aur are smaller that the pressure scale height. In this case, its EMD can be interpreted in terms of a population of coronal loops each of them having $\operatorname{EM}(T) \propto T^{3}$. This slope is significantly steeper than the value $\alpha=1.5$ obtained for loops with constant cross-section and uniform heating, which well describes the ascending slope of the EMD derived for the solar corona as a whole (Orlando et al. 2000; Peres et al. 2000). This imples that the dominant structures with $T_{\max } \gtrsim 10^{7} \mathrm{~K}$ in the corona of SU Aur are different from the solar ones, and are characterized by an excess of emission measure at higher temperatures. A steeper slope can be attained if the heating is concentrated at the loop footpoints, resulting in a lower temperature gradient along the loop (Testa et al. 2005), or in the case of loops expanding with height, implying more emission measure at higher temperatures (Schrijver et al. 1989; Sim \& Jordan 2003). Both mechanisms may also be at work.

Finally, our line-based analysis for SU Aur confirms the higher iron abundance and the lower $\mathrm{Ne} / \mathrm{Fe}$ ratio found by Telleschi et al. (2007b) for this and other G-type stars, with respect to later-type PMS and older active stars.

Acknowledgements. We thank the referee for his/her useful comments which helped improving the paper. E.F., L.S. and R.P. acknowledge financial 
contribution from Ministero dell'Università e della Ricerca (MiUR) and from contract ASI-INAF I/023/05/0. M.A. acknowledges support from a Swiss National Science Foundation Professorship (PP002-110504). This work is based on observations obtained with XMM-Newton, an ESA science mission with instruments and contributions directly funded by ESA Member States and NASA. This publication makes use of data products from the Two Micron All Sky Survey, which is a joint project of the University of Massachusetts and the Infrared Processing and Analysis Center/California Institute of Technology, funded by the National Aeronautics and Space Administration and the National Science Foundation.

\section{References}

Alexander, D. M., Bauer, F. E., Brandt, W. N., et al. 2003, AJ, 126, 539 Anders, E., \& Grevesse, N. 1989, Geochim. Cosmochim. Acta, 53, 197 Argiroffi, C., Maggio, A., \& Peres, G. 2003, A\&A, 404, 1033

Argiroffi, C., Maggio, A., Peres, G., Stelzer, B., \& Neuhäuser, R. 2005, A\&A, 439, 1149

Argiroffi, C., Maggio, A., \& Peres, G. 2007, A\&A, 465, L5

Budding, E., Erdem, A., Çiçek, C., et al. 2004, A\&A, 417, 263

Calvet, N., \& Gullbring, E. 1998, ApJ, 509, 802

Damiani, F., Micela, G., Sciortino, S., \& Harnden, Jr., F. R. 1995, ApJ, 446, 331

Damiani, F., Maggio, A., Micela, G., \& Sciortino, S. 1997, ApJ, 483, 350

DeWarf, L. E., Sepinsky, J. F., Guinan, E. F., Ribas, I., \& Nadalin, I. 2003, ApJ, 590,357

ESA. 1997, The Hipparcos and Tycho catalogues, ESA SP-1200

Favata, F., \& Micela, G. 2003, Space Sci. Rev., 108, 577

Feigelson, E. D., \& DeCampli, W. M. 1981, ApJ, 243, L89

Feigelson, E. D., \& Montmerle, T. 1999, ARA\&A, 37, 363

Feigelson, E. D., Gaffney, J. A. I., Garmire, G., Hillenbrand, L. A., \& Townsley, L. 2003, ApJ, 584, 911

Flaccomio, E., Damiani, F., Micela, G., et al. 2003, ApJ, 582, 398

Franciosini, E., Pallavicini, R., \& Sanz-Forcada, J. 2006, A\&A, 446, 501

Franciosini, E., Pillitteri, I., Stelzer, B., et al. 2007, A\&A, 468, 485

Güdel, M., Briggs, K. R., Arzner, K., et al. 2007a, A\&A, 468, 353

Güdel, M., Skinner, S. L., Mel'nikov, S. Y., et al. 2007b, A\&A, 468, 529

Günther, H. M., Liefke, C., Schmitt, J. H. M. M., Robrade, J., \& Ness, J.-U. 2006, A\&A, 459, L29

Jardine, M., Collier Cameron, A., Donati, J.-F., Gregory, S. G., \& Wood, K. 2006, MNRAS, 367, 917

Jones, B. F., \& Herbig, G. H. 1979, AJ, 84, 1872

Kashyap, V., \& Drake, J. J. 1998, ApJ, 503, 450
Kashyap, V., \& Drake, J. J. 2000, Bull. Astron. Soc. India, 28, 475

Kastner, J. H., Huenemorder, D. P., Schulz, N. S., Canizares, C. R., \& Weintraub,

D. A. 2002, ApJ, 567, 434

Maggio, A., \& Peres, G. 1996, A\&A, 306, 563

Mazzotta, P., Mazzitelli, G., Colafrancesco, S., \& Vittorio, N. 1998, A\&AS, 133, 403

Neuhäuser, R., Sterzik, M. F., Schmitt, J. H. M. M., Wichmann, R., \& Krautter, J. 1995, A\&A, 297, 391

Orlando, S., Peres, G., \& Reale, F. 2000, ApJ, 528, 524

Oudmaijer, R. D., van der Veen, W. E. C. J., Waters, L. B. F. M., et al. 1992, A\&AS, 96, 625

Peres, G., Orlando, S., Reale, F., Rosner, R., \& Hudson, H. 2000, ApJ, 528, 537

Peres, G., Orlando, S., Reale, F., \& Rosner, R. 2001, ApJ, 563, 1045

Preibisch, T., Kim, Y.-C., Favata, F., et al. 2005, ApJS, 160, 401

Randich, S., \& Schmitt, J. H. M. M. 1995, A\&A, 298, 115

Robrade, J., \& Schmitt, J. H. H. M. 2006, A\&A, 449, 737

Sanz-Forcada, J., Maggio, A., \& Micela, G. 2003, A\&A, 408, 1087

Scelsi, L., Maggio, A., Peres, G., \& Gondoin, P. 2004, A\&A, 413, 643

Scelsi, L., Maggio, A., Peres, G., \& Pallavicini, R. 2005, A\&A, 432, 671

Scelsi, L., Maggio, A., Micela, G., et al. 2007, A\&A, 468, 405

Schmitt, J. H. M. M., Robrade, J., Ness, J.-U., Favata, F., \& Stelzer, B. 2005, A\&A, 432, L35

Schrijver, C. J., Lemen, J. R., \& Mewe, R. 1989, ApJ, 341, 484

Serio, S., Reale, F., Jakimiec, J., Sylwester, B., \& Sylwester, J. 1991, A\&A, 241, 197

Sim, S. A., \& Jordan, C. 2003, MNRAS, 346, 846

Skinner, S. L., \& Walter, F. M. 1998, ApJ, 509, 761

Skrutskie, M. F., Cutri, R. M., Stiening, R., et al. 2006, AJ, 131, 1163

Smith, K., Audard, M., Güdel, M., Skinner, S., \& Pallavicini, R. 2005, in Cool Stars, Stellar Systems, and the Sun: 13th Cambridge Workshop, ed. F. Favata, \& J. H. M. M. Schmitt, ESA-SP, 560, 971

Stassun, K. G., Ardila, D. R., Barsony, M., Basri, G., \& Mathieu, R. D. 2004, AJ, 127, 3537

Stelzer, B., \& Neuhäuser, R. 2001, A\&A, 377, 538

Stelzer, B., \& Schmitt, J. H. M. M. 2004, A\&A, 418, 687

Telleschi, A., Güdel, M., Briggs, K. R., Audard, M., \& Palla, F. 2007a, A\&A, 468,425

Telleschi, A., Güdel, M., Briggs, K. R., Audard, M., \& Scelsi, L. 2007b, A\&A, 468,443

Telleschi, A., Güdel, M., Briggs, K. R., et al. 2007c, A\&A, 468, 541

Testa, P., Peres, G., \& Reale, F. 2005, ApJ, 622, 695

Tozzi, P., Rosati, P., Nonino, M., et al. 2001, ApJ, 562, 42

Wichmann, R., Krautter, J., Schmitt, J. H. M. M., et al. 1996, A\&A, 312, 439 
E. Franciosini et al.: XMM-Newton observation of SU Aurigae and the surrounding region, Online Material $p 1$

\section{Online Material}


E. Franciosini et al.: XMM-Newton observation of SU Aurigae and the surrounding region, Online Material p 2

Table 2. Strongest lines measured in the RGS spectrum of SU Aur. Only lines with $S / N \geq 1.5$ are listed. Asterisks mark the lines used for the EM reconstruction. $\lambda_{\mathrm{obs}}$ is the measured wavelenght, $\lambda_{\text {pred }}$ is the theoretical wavelenght of the transition, and $T_{\max }$ is the temperature of maximum emissivity in $\mathrm{K}$.

\begin{tabular}{|c|c|c|c|c|c|c|}
\hline & $\begin{array}{l}\lambda_{\text {obs }} \\
(\AA)\end{array}$ & Total counts & Ion & $\begin{array}{r}\lambda_{\text {pred }} \\
(\AA)\end{array}$ & $\begin{array}{l}\text { Transition } \\
(\text { upper } \rightarrow \text { lower })\end{array}$ & $\log T_{\mathrm{max}}$ \\
\hline \multirow[t]{11}{*}{ * } & \multirow[t]{2}{*}{$6.19 \pm 0.03$} & \multirow[t]{2}{*}{$27 \pm 13$} & Si XIV & 6.18 & $2 \mathrm{p}^{2} \mathrm{P}_{3 / 2} \rightarrow 1 \mathrm{~s}^{2} \mathrm{~S}_{1 / 2}$ & 7.2 \\
\hline & & & Si XIV & 6.19 & $2 \mathrm{p}^{2} \mathrm{P}_{1 / 2} \rightarrow 1 \mathrm{~s}^{2} \mathrm{~S}_{1 / 2}$ & 7.2 \\
\hline & \multirow[t]{2}{*}{$6.71 \pm 0.04$} & \multirow[t]{2}{*}{$23 \pm 13$} & Si XIII & 6.65 & $1 \mathrm{~s} 2 \mathrm{p}^{1} \mathrm{P}_{1} \rightarrow 1 \mathrm{~s}^{2}{ }^{1} \mathrm{~S}_{0}$ & 7.0 \\
\hline & & & Si XIII & 6.69 & $1 \mathrm{~s} 2 \mathrm{p}^{3} \mathrm{P}_{1} \rightarrow 1 \mathrm{~s}^{2}{ }^{1} \mathrm{~S}_{0}$ & 7.0 \\
\hline & \multirow[t]{2}{*}{$7.11 \pm 0.02$} & \multirow[t]{2}{*}{$25 \pm 13$} & $\operatorname{Mg}$ XII & 7.11 & $3 \mathrm{p}^{2} \mathrm{P}_{3 / 2} \rightarrow 1 \mathrm{~s}^{2} \mathrm{~S}_{1 / 2}$ & 7.0 \\
\hline & & & $\operatorname{Mg}$ XII & 7.11 & $3 \mathrm{p}^{2} \mathrm{P}_{1 / 2} \rightarrow 1 \mathrm{~s}^{2} \mathrm{~S}_{1 / 2}$ & 7.0 \\
\hline & \multirow[t]{3}{*}{$7.86 \pm 0.03$} & \multirow[t]{3}{*}{$25 \pm 13$} & $\mathrm{Mg}$ XI & 7.85 & $1 \mathrm{~s} 3 \mathrm{p}^{1} \mathrm{P}_{1} \rightarrow 1 \mathrm{~s}^{2}{ }^{1} \mathrm{~S}_{0}$ & 6.8 \\
\hline & & & $\mathrm{Fe} \mathrm{XXIII}$ & 7.90 & $1 s^{2} 2 s_{5} d^{1} D_{2} \rightarrow 1 s^{2} 2 s 2{ }^{1} P_{1}$ & 7.2 \\
\hline & & & Al XII & 7.87 & $1 \mathrm{~s} 2 \mathrm{~s}^{3} \mathrm{~S}_{1} \rightarrow 1 \mathrm{~s}^{2}{ }^{1} \mathrm{~S}_{0}$ & 6.9 \\
\hline & \multirow[t]{2}{*}{$8.24 \pm 0.03$} & \multirow[t]{2}{*}{$29 \pm 15$} & Fe XXIV & 8.23 & $1 \mathrm{~s}^{2} 4 \mathrm{~d}^{2} \mathrm{D}_{3 / 2} \rightarrow 1 \mathrm{~s}^{2} 2 \mathrm{p}^{2} \mathrm{P}_{1 / 2}$ & 7.3 \\
\hline & & & $\mathrm{Ni}$ XXIII & 8.27 & $1 \mathrm{~s}^{2} 2 \mathrm{~s}^{2} 2 \mathrm{p} 4 \mathrm{~d}^{3} \mathrm{P}_{1} \rightarrow 1 \mathrm{~s}^{2} 2 \mathrm{~s}^{2} 2 \mathrm{p}^{2}{ }^{3} \mathrm{P}_{0}$ & 7.1 \\
\hline \multirow[t]{20}{*}{$*$} & \multirow[t]{2}{*}{$8.42 \pm 0.02$} & \multirow[t]{2}{*}{$49 \pm 16$} & Mg XII & 8.42 & $2 \mathrm{p}^{2} \mathrm{P}_{3 / 2} \rightarrow 1 \mathrm{~s}^{2} \mathrm{~S}_{1 / 2}$ & 7.0 \\
\hline & & & Mg XII & 8.42 & $2 \mathrm{p}^{2} \mathrm{P}_{1 / 2} \rightarrow 1 \mathrm{~s}^{2} \mathrm{~S}_{1 / 2}$ & 7.0 \\
\hline & \multirow[t]{4}{*}{$8.65 \pm 0.04$} & \multirow[t]{4}{*}{$26 \pm 14$} & Fe XXIII & 8.62 & $1 \mathrm{~s}^{2} 2 \mathrm{~s} 4 \mathrm{~d}^{3} \mathrm{D}_{3} \rightarrow 1 \mathrm{~s}^{2} 2 \mathrm{~s} 2 \mathrm{p}^{3} \mathrm{P}_{2}$ & 7.2 \\
\hline & & & Fe XXI & 8.61 & $1 \mathrm{~s}^{2} 2 \mathrm{~s}^{2} 2 \mathrm{p}_{1 / 2} 5 \mathrm{~d}_{3 / 2} \rightarrow 1 \mathrm{~s}^{2} 2 \mathrm{~s}^{2} 2 \mathrm{p}^{2}{ }^{3} \mathrm{P}_{1}$ & 7.1 \\
\hline & & & Fe XXI & 8.64 & $1 \mathrm{~s}^{2} 2 \mathrm{~s}^{2} 2 \mathrm{p} 5 \mathrm{~d}^{3} \mathrm{P}_{2} \rightarrow 1 \mathrm{~s}^{2} 2 \mathrm{~s}^{2} 2 \mathrm{p}^{2}{ }^{3} \mathrm{P}_{1}$ & 7.0 \\
\hline & & & Fe XXI & 8.66 & $1 s^{2} 2 s^{2} 2 p 5 d^{3} F_{3} \rightarrow 1 s^{2} 2 s^{2} 2 p^{2}{ }^{3} P_{2}$ & 7.0 \\
\hline & \multirow[t]{2}{*}{$8.92 \pm 0.03$} & \multirow[t]{2}{*}{$21 \pm 13$} & Fe XXIII & 8.91 & $1 \mathrm{~s}^{2} 2 \mathrm{~s} 4 \mathrm{~s}^{1} \mathrm{~S}_{0} \rightarrow 1 \mathrm{~s}^{2} 2 \mathrm{~s} 2 \mathrm{p}^{1} \mathrm{P}_{1}$ & 7.2 \\
\hline & & & Fe XXI & 8.93 & $1 \mathrm{~s}^{2} 2 \mathrm{~s} 2 \mathrm{p}^{2} 5 \mathrm{~s} \rightarrow 1 \mathrm{~s}^{2} 2 \mathrm{~s}^{2} \mathrm{p}^{3}{ }^{3} \mathrm{D}_{1}$ & 7.0 \\
\hline & \multirow[t]{3}{*}{$9.21 \pm 0.02$} & \multirow[t]{3}{*}{$43 \pm 15$} & $\operatorname{Mg}$ XI & 9.23 & $1 \mathrm{~s} 2 \mathrm{p}^{3} \mathrm{P}_{1} \rightarrow 1 \mathrm{~s}^{2}{ }^{1} \mathrm{~S}_{0}$ & 6.8 \\
\hline & & & Fe XXI & 9.19 & $1 \mathrm{~s}^{2} 2 \mathrm{~s} 2 \mathrm{p}_{1 / 2}^{2} 4 \mathrm{p}_{3 / 2} \rightarrow 1 \mathrm{~s}^{2} 2 \mathrm{~s}^{2} 2 \mathrm{p}^{2}{ }^{3} \mathrm{P}_{0}$ & 7.1 \\
\hline & & & Fe XX & 9.19 & $1 s^{2} 2 s_{2} p_{1 / 2} 2 p_{3 / 2}^{2} 4 p_{3 / 2} \rightarrow 2 s^{2} 2 p^{3}{ }^{4} S_{3 / 2}$ & 7.0 \\
\hline & $9.35 \pm 0.03$ & $24 \pm 14$ & Mg XI & 9.31 & $1 \mathrm{~s} 2 \mathrm{~s}{ }^{3} \mathrm{~S}_{1} \rightarrow 1 \mathrm{~s}^{2}{ }^{1} \mathrm{~S}_{0}$ & 6.8 \\
\hline & $9.47 \pm 0.02$ & $29 \pm 15$ & Fe XXI & 9.48 & $1 s^{2} 2 s^{2} 2 p 4 d^{3} P_{1} \rightarrow 1 s^{2} 2 s^{2} 2 p^{2}{ }^{3} P_{0}$ & 7.0 \\
\hline & & & $\mathrm{NeX}$ & 9.48 & $5 \mathrm{p}^{2} \mathrm{P}_{3 / 2} \rightarrow 1 \mathrm{~s}^{2} \mathrm{~S}_{1 / 2}$ & 6.8 \\
\hline & $9.71 \pm 0.02$ & $20 \pm 14$ & $\mathrm{NeX}$ & 9.71 & $4 \mathrm{p}^{2} \mathrm{P}_{3 / 2} \rightarrow 1 \mathrm{~s}^{2} \mathrm{~S}_{1 / 2}$ & 6.8 \\
\hline & & & $\mathrm{NeX}$ & 9.71 & $4 \mathrm{p}^{2} \mathrm{P}_{1 / 2} \rightarrow 1 \mathrm{~s}^{2} \mathrm{~S}_{1 / 2}$ & 6.8 \\
\hline & & & Fexx & 9.73 & $1 s^{2} 2 s_{2} p_{1 / 2}^{2} 2 p_{3 / 2} 4 p_{3 / 2} \rightarrow 2 s^{2} 2 p^{3}{ }^{4} S_{3 / 2}$ & 7.0 \\
\hline & & & Fe XIX & 9.70 & $2 s^{2} 2 p^{3}\left({ }^{2} \mathrm{D}\right) 5 \mathrm{~d}^{3} \mathrm{D}_{3} \rightarrow 2 \mathrm{~s}^{2} 2 \mathrm{p}^{4}{ }^{3} \mathrm{P}_{2}$ & 6.9 \\
\hline & & & Fe Xx & 9.73 & $1 \mathrm{~s}^{2} 2 \mathrm{~s} 2 \mathrm{p}_{1 / 2}^{2} 2 \mathrm{p}_{3 / 2} 4 \mathrm{p}_{3 / 2} \rightarrow 2 \mathrm{~s}^{2} 2 \mathrm{p}^{3}{ }^{4} \mathrm{~S}_{3 / 2}$ & 7.0 \\
\hline & $10.06 \pm 0.02$ & $32 \pm 14$ & Fe XX & 10.05 & $1 s^{2} 2 s^{2} 2 p_{1 / 2} 2 p_{3 / 2} 4 d_{3 / 2} \rightarrow 2 s^{2} 2 p^{3}{ }^{4} S_{3 / 2}$ & 7.0 \\
\hline & & & $\mathrm{Fe} x \mathrm{X}$ & 10.06 & $1 \mathrm{~s}^{2} 2 \mathrm{~s}^{2} 2 \mathrm{p}_{1 / 2} 2 \mathrm{p}_{3 / 2} 4 \mathrm{~d}_{3 / 2} \rightarrow 2 \mathrm{~s}^{2} 2 \mathrm{p}^{3}{ }^{4} \mathrm{~S}_{3 / 2}$ & 7.0 \\
\hline$*$ & $10.20 \pm 0.02$ & $39 \pm 15$ & $\mathrm{Ne} \mathrm{X}$ & 10.24 & $3 \mathrm{p}^{2} \mathrm{P}_{3 / 2} \rightarrow 1 \mathrm{~s}^{2} \mathrm{~S}_{1 / 2}$ & 6.8 \\
\hline & & & $\mathrm{Ne} \mathbf{X}$ & 10.24 & $3 \mathrm{p}^{2} \mathrm{P}_{1 / 2} \rightarrow 1 \mathrm{~s}^{2} \mathrm{~S}_{1 / 2}$ & 6.8 \\
\hline * & $10.63 \pm 0.01$ & $43 \pm 15$ & Fe XXIV & 10.62 & $1 \mathrm{~s}^{2} 3 \mathrm{p}^{2} \mathrm{P}_{3 / 2} \rightarrow 1 \mathrm{~s}^{2} 2 \mathrm{~s}^{2} \mathrm{~S}_{1 / 2}$ & 7.3 \\
\hline & & & Fe XXIV & 10.66 & $1 \mathrm{~s}^{2} 3 \mathrm{p}^{2} \mathrm{P}_{1 / 2} \rightarrow 1 \mathrm{~s}^{2} 2 \mathrm{~s}^{2} \mathrm{~S}_{1 / 2}$ & 7.3 \\
\hline & & & Fe XIX & 10.65 & $2 \mathrm{~s}^{2} 2 \mathrm{p}^{3}\left({ }^{2} \mathrm{D}\right) 4 \mathrm{~d}^{3} \mathrm{D}_{3} \rightarrow 2 \mathrm{~s}^{2} 2 \mathrm{p}^{4}{ }^{3} \mathrm{P}_{2}$ & 6.9 \\
\hline & & & Fe XIX & 10.64 & $2 \mathrm{~s}^{2} 2 \mathrm{p}^{3}\left({ }^{2} \mathrm{D}\right) 4 \mathrm{~d}^{3} \mathrm{P}_{2} \rightarrow 2 \mathrm{~s}^{2} 2 \mathrm{p}^{4}{ }^{3} \mathrm{P}_{2}$ & 6.9 \\
\hline * & $11.05 \pm 0.03$ & $22 \pm 13$ & Fe XXIV & 11.03 & $1 \mathrm{~s}^{2} 3 \mathrm{~d}^{2} \mathrm{D}_{3 / 2} \rightarrow 1 \mathrm{~s}^{2} 2 \mathrm{p}^{2} \mathrm{P}_{1 / 2}$ & 7.3 \\
\hline & & & Fe XXIII & 11.02 & $1 s^{2} 2 s 3 p^{3} P_{1} \rightarrow 1 s^{2} 2 s^{2}{ }^{1} S_{0}$ & 7.2 \\
\hline & $11.13 \pm 0.03$ & $21 \pm 13$ & Fe XVII & 11.13 & $2 \mathrm{~s}^{2} 2 \mathrm{p}^{5}\left({ }^{2} \mathrm{P}\right) 5 \mathrm{~d}^{1} \mathrm{P}_{1} \rightarrow 2 \mathrm{~s}^{2} 2 \mathrm{p}^{6}{ }^{1} \mathrm{~S}_{0}$ & 6.8 \\
\hline & $11.36 \pm 0.02$ & $28 \pm 13$ & Fe XVIII & 11.33 & $2 s^{2} 2 p^{4}\left({ }^{1} \mathrm{D}\right) 4 d^{2} D_{5 / 2} \rightarrow 2 s^{2} 2 p^{5}{ }^{2} P_{3 / 2}$ & 6.9 \\
\hline & & & Fe XVIII & 11.33 & $2 \mathrm{~s}^{2} 2 \mathrm{p}^{4}\left({ }^{1} \mathrm{D}\right) 4 \mathrm{~d}^{2} \mathrm{P}_{3 / 2} \rightarrow 2 \mathrm{~s}^{2} 2 \mathrm{p}^{5}{ }^{2} \mathrm{P}_{3 / 2}$ & 6.9 \\
\hline & & & Fe XVIII & 11.33 & $2 \mathrm{~s}^{2} 2 \mathrm{p}^{4}\left({ }^{1} \mathrm{D}\right) 4 \mathrm{~d}^{2} \mathrm{~S}_{1 / 2} \rightarrow 2 \mathrm{~s}^{2} 2 \mathrm{p}^{5}{ }^{2} \mathrm{P}_{3 / 2}$ & 6.9 \\
\hline & & & Fe XXIII & 11.34 & $1 \mathrm{~s}^{2} 2 \mathrm{~s} 3 \mathrm{~d}^{3} \mathrm{D}_{2} \rightarrow 1 \mathrm{~s}^{2} 2 \mathrm{~s} 2 \mathrm{p}^{3} \mathrm{P}_{1}$ & 7.2 \\
\hline & & & Ni XXI & 11.32 & $2 s^{2} 2 p^{3}\left({ }^{2} \mathrm{D}\right) 3 d^{3} D_{3} \rightarrow 2 s^{2} 2 p^{4}{ }^{3} P_{2}$ & 7.0 \\
\hline & $11.46 \pm 0.02$ & $25 \pm 13$ & Fe XXIV & 11.43 & $1 \mathrm{~s}^{2} 3 \mathrm{~s}^{2} \mathrm{~S}_{1 / 2} \rightarrow 1 \mathrm{~s}^{2} 2 \mathrm{p}^{2} \mathrm{P}_{3 / 2}$ & 7.3 \\
\hline & & & Fe XXII & 11.49 & $1 \mathrm{~s}^{2} 2 \mathrm{~s}_{2} \mathrm{p}_{1 / 2} 3 \mathrm{p}_{3 / 2} \rightarrow 1 \mathrm{~s}^{2} 2 \mathrm{~s}^{2} 2 \mathrm{p}^{2} \mathrm{P}_{1 / 2}$ & 7.1 \\
\hline & & & Fe XXIII & 11.46 & $1 \mathrm{~s}^{2} 2 \mathrm{~s} 3 \mathrm{~d}^{3} \mathrm{D}_{3} \rightarrow 1 \mathrm{~s}^{2} 2 \mathrm{~s} 2 \mathrm{p}^{3} \mathrm{P}_{2}$ & 7.2 \\
\hline * & $11.75 \pm 0.02$ & $40 \pm 13$ & Fe XXIII & 11.74 & $1 \mathrm{~s}^{2} 2 \mathrm{~s} 3 \mathrm{~d}^{1} \mathrm{D}_{2} \rightarrow 1 \mathrm{~s}^{2} 2 \mathrm{~s} 2 \mathrm{p}^{1} \mathrm{P}_{1}$ & 7.2 \\
\hline & & & Fe XXII & 11.77 & $1 s^{2} 2 s^{2} 3 d^{2} D_{3 / 2} \rightarrow 1 s^{2} 2 s^{2} 2 p^{2} P_{1 / 2}$ & 7.1 \\
\hline
\end{tabular}


E. Franciosini et al.: XMM-Newton observation of SU Aurigae and the surrounding region, Online Material p 3

Table 2. continued.

\begin{tabular}{|c|c|c|c|c|c|c|}
\hline & $\begin{array}{l}\lambda_{\text {obs }} \\
(\AA)\end{array}$ & Total counts & Ion & $\begin{array}{r}\lambda_{\text {pred }} \\
(\AA)\end{array}$ & $\begin{array}{l}\text { Transition } \\
\text { (upper } \rightarrow \text { lower })\end{array}$ & $\log T_{\max }$ \\
\hline & \multirow[t]{4}{*}{$11.94 \pm 0.03$} & \multirow[t]{4}{*}{$19 \pm 12$} & Fe XXII & 11.98 & $1 s^{2} 2 s^{2} 3 d^{2} D_{3 / 2} \rightarrow 1 s^{2} 2 s^{2} 2 p^{2} P_{3 / 2}$ & 7.1 \\
\hline & & & Fe XXI & 11.98 & $1 \mathrm{~s}^{2} 2 \mathrm{~s} 2 \mathrm{p}_{1 / 2}^{2} 3 \mathrm{p}_{3 / 2} \rightarrow 1 \mathrm{~s}^{2} 2 \mathrm{~s}^{2} 2 \mathrm{p}^{2}{ }^{3} \mathrm{P}_{0}$ & 7.0 \\
\hline & & & Fe XXII & 11.93 & $1 s^{2} 2 s^{2} 3 d^{2} D_{5 / 2} \rightarrow 1 s^{2} 2 s^{2} 2 p^{2} P_{3 / 2}$ & 7.1 \\
\hline & & & Fe XXI & 11.95 & $1 \mathrm{~s}^{2} 2 \mathrm{~s} 2 \mathrm{p}_{1 / 2} 2 \mathrm{p}_{3 / 2} 3 \mathrm{p}_{3 / 2} \rightarrow 1 \mathrm{~s}^{2} 2 \mathrm{~s}^{2} 2 \mathrm{p}^{2}{ }^{3} \mathrm{P}_{0}$ & 7.0 \\
\hline \multirow[t]{4}{*}{$*$} & \multirow[t]{4}{*}{$12.16 \pm 0.01$} & \multirow[t]{4}{*}{$100 \pm 17$} & $\mathrm{NeX}$ & 12.13 & $2 \mathrm{p}^{2} \mathrm{P}_{3 / 2} \rightarrow 1 \mathrm{~s}^{2} \mathrm{~S}_{1 / 2}$ & 6.8 \\
\hline & & & $\operatorname{NeX}$ & 12.14 & $2 \mathrm{p}^{2} \mathrm{P}_{1 / 2} \rightarrow 1 \mathrm{~s}^{2} \mathrm{~S}_{1 / 2}$ & 6.8 \\
\hline & & & Fe XVII & 12.12 & $2 \mathrm{~s}^{2} 2 \mathrm{p}^{5}\left({ }^{2} \mathrm{P}\right) 4 \mathrm{~d}^{1} \mathrm{P}_{1} \rightarrow 2 \mathrm{~s}^{2} 2 \mathrm{p}^{6}{ }^{1} \mathrm{~S}_{0}$ & 6.8 \\
\hline & & & Fe XXIII & 12.16 & $1 s^{2} 2 s 3 s^{1} S_{0} \rightarrow 1 s^{2} 2 s 2 p^{1} P_{1}$ & 7.2 \\
\hline \multirow[t]{5}{*}{ * } & & $54 \pm 15$ & Fe XXI & 12.28 & $1 s^{2} 2 s^{2} 2 p 3 d^{3} D_{1} \rightarrow 1 s^{2} 2 s^{2} 2 p^{2}{ }^{3} P_{0}$ & 7.0 \\
\hline & \multirow[t]{4}{*}{$12.68 \pm 0.02$} & \multirow[t]{4}{*}{$26 \pm 13$} & Fe XXI & 12.65 & $1 \mathrm{~s}^{2} 2 \mathrm{~s} 2 \mathrm{p}_{1 / 2} 2 \mathrm{p}_{3 / 2} 3 \mathrm{~d}_{5 / 2} \rightarrow 1 \mathrm{~s}^{2} 2 \mathrm{~s} 2 \mathrm{p}^{3}{ }^{3} \mathrm{~S}_{1}$ & 7.0 \\
\hline & & & Ni XIX & 12.66 & $2 \mathrm{~s}^{2} 2 \mathrm{p}^{5}\left({ }^{2} \mathrm{P}\right) 3 \mathrm{~d}^{3} \mathrm{D}_{1} \rightarrow 2 \mathrm{~s}^{2} 2 \mathrm{p}^{6}{ }^{1} \mathrm{~S}_{0}$ & 6.9 \\
\hline & & & Fe XVII & 12.70 & $2 s^{2} 2 p^{5}\left({ }^{2} \mathrm{P}\right) 4 s^{3} \mathrm{P}_{1} \rightarrow 2 \mathrm{~s}^{2} 2 \mathrm{p}^{6}{ }^{1} \mathrm{~S}_{0}$ & 6.8 \\
\hline & & & Fe XXII & 12.63 & $1 \mathrm{~s}^{2} 2 \mathrm{~s} 2 \mathrm{p}_{1 / 2} 3 \mathrm{~d}_{5 / 2} \rightarrow 1 \mathrm{~s}^{2} 2 \mathrm{~s} 2 \mathrm{p}^{2}{ }^{2} \mathrm{P}_{3 / 2}$ & 7.1 \\
\hline \multirow[t]{16}{*}{$*$} & \multirow[t]{3}{*}{$12.80 \pm 0.02$} & \multirow[t]{3}{*}{$36 \pm 13$} & $\mathrm{Fe} x \mathrm{x}$ & 12.82 & $1 \mathrm{~s}^{2} 2 \mathrm{~s}^{2} 2 \mathrm{p}_{1 / 2} 2 \mathrm{p}_{3 / 2} 3 \mathrm{~d}_{3 / 2} \rightarrow 2 \mathrm{~s}^{2} 2 \mathrm{p}^{3}{ }^{4} \mathrm{~S}_{3 / 2}$ & 7.0 \\
\hline & & & Fe XXII & 12.75 & $1 \mathrm{~s}^{2} 2 \mathrm{~s} 2 \mathrm{p}_{1 / 2} 3 \mathrm{~s} \rightarrow 1 \mathrm{~s}^{2} 2 \mathrm{~s} 2 \mathrm{p}^{2}{ }^{2} \mathrm{D}_{3 / 2}$ & 7.1 \\
\hline & & & Fe XXI & 12.82 & $1 \mathrm{~s}^{2} 2 \mathrm{~s} 2 \mathrm{p}_{1 / 2} 2 \mathrm{p}_{3 / 2} 3 \mathrm{~d}_{5 / 2} \rightarrow 1 \mathrm{~s}^{2} 2 \mathrm{~s} 2 \mathrm{p}^{3}{ }^{3} \mathrm{D}_{1}$ & 7.0 \\
\hline & \multirow[t]{4}{*}{$12.95 \pm 0.01$} & \multirow[t]{4}{*}{$46 \pm 13$} & Fe XX & 12.97 & $1 s^{2} 2 s^{2} 2 p_{1 / 2} 2 p_{3 / 2} 3 d_{3 / 2} \rightarrow 2 s^{2} 2 p^{3}{ }^{4} S_{3 / 2}$ & 7.0 \\
\hline & & & Fe Xx & 12.91 & $1 \mathrm{~s}^{2} 2 \mathrm{~s}^{2} 2 \mathrm{p}_{1 / 2} 2 \mathrm{p}_{3 / 2} 3 \mathrm{~d}_{5 / 2} \rightarrow 2 \mathrm{~s}^{2} 2 \mathrm{p}^{3}{ }^{4} \mathrm{~S}_{3 / 2}$ & 7.0 \\
\hline & & & Fe XXII & 12.95 & $1 \mathrm{~s}^{2} 2 \mathrm{~s} 2 \mathrm{p}_{1 / 2} 3 \mathrm{~s} \rightarrow 1 \mathrm{~s}^{2} 2 \mathrm{~s}^{2} \mathrm{p}^{2}{ }^{2} \mathrm{~S}_{1 / 2}$ & 7.1 \\
\hline & & & Fe XIX & 12.93 & $1 \mathrm{~s}^{2} 2 \mathrm{~s} 2 \mathrm{p}_{1 / 2} 2 \mathrm{p}_{3 / 2}^{3} 3 \mathrm{p}_{3 / 2} \rightarrow 2 \mathrm{~s}^{2} 2 \mathrm{p}^{4}{ }^{3} \mathrm{P}_{2}$ & 6.9 \\
\hline & \multirow[t]{5}{*}{$13.25 \pm 0.02$} & \multirow[t]{5}{*}{$25 \pm 12$} & Fe XXI & 13.25 & $1 s^{2} 2 s^{2} 2 p_{1 / 2} 3 s \rightarrow 1 s^{2} 2 s^{2} 2 p^{2}{ }^{3} P_{2}$ & 7.0 \\
\hline & & & Fe XXII & 13.24 & $1 s^{2} 2 s^{2} 3 p^{2} P_{1 / 2} \rightarrow 1 s^{2} 2 s 2 p^{2}{ }^{2} D_{3 / 2}$ & 7.1 \\
\hline & & & Fe Xx & 13.27 & $1 \mathrm{~s}^{2} 2 \mathrm{~s}^{2} 2 \mathrm{p}_{1 / 2} 2 \mathrm{p}_{3 / 2} 3 \mathrm{~d}_{3 / 2} \rightarrow 2 \mathrm{~s}^{2} 2 \mathrm{p}^{3}{ }^{2} \mathrm{P}_{1 / 2}$ & 7.0 \\
\hline & & & $\mathrm{Fe} X \mathrm{X}$ & 13.27 & $1 \mathrm{~s}^{2} 2 \mathrm{~s}^{2} 2 \mathrm{p}_{1 / 2} 2 \mathrm{p}_{3 / 2} 3 \mathrm{~d}_{5 / 2} \rightarrow 2 \mathrm{~s}^{2} 2 \mathrm{p}^{3}{ }^{2} \mathrm{P}_{1 / 2}$ & 7.0 \\
\hline & & & Fe XIX & 13.27 & $2 \mathrm{~s}^{2} 2 \mathrm{p}^{3}\left({ }^{2} \mathrm{P}\right) 3 \mathrm{~d}^{3} \mathrm{D}_{3} \rightarrow 2 \mathrm{~s}^{2} 2 \mathrm{p}^{4}{ }^{3} \mathrm{P}_{2}$ & 6.9 \\
\hline & \multirow[t]{4}{*}{$13.34 \pm 0.03$} & \multirow[t]{4}{*}{$18 \pm 12$} & Fe XX & 13.35 & $1 \mathrm{~s}^{2} 2 \mathrm{~s}^{2} 2 \mathrm{p}_{1 / 2}^{2} 3 \mathrm{~d}_{3 / 2} \rightarrow 2 \mathrm{~s}^{2} 2 \mathrm{p}^{3}{ }^{2} \mathrm{D}_{3 / 2}$ & 7.0 \\
\hline & & & $\mathrm{Fe} x \mathrm{x}$ & 13.39 & $2 \mathrm{~s} 2 \mathrm{p}^{3}\left({ }^{5} \mathrm{~S}\right) 3 \mathrm{~d}^{4} \mathrm{D}_{7 / 2} \rightarrow 2 \mathrm{~s} 2 \mathrm{p}^{4}{ }^{4} \mathrm{P}_{5 / 2}$ & 6.9 \\
\hline & & & Fe XVIII & 13.32 & $2 \mathrm{~s} 2 \mathrm{p}^{5}\left({ }^{3} \mathrm{P}\right) 3 \mathrm{p}^{4} \mathrm{P}_{5 / 2} \rightarrow 2 \mathrm{~s}^{2} 2 \mathrm{p}^{5}{ }^{2} \mathrm{P}_{3 / 2}$ & 6.9 \\
\hline & & & Fe XVIII & 13.36 & $2 \mathrm{~s} 2 \mathrm{p}^{5}\left({ }^{3} \mathrm{P}\right) 3 \mathrm{p}^{2} \mathrm{P}_{3 / 2} \rightarrow 2 \mathrm{~s}^{2} 2 \mathrm{p}^{5}{ }^{2} \mathrm{P}_{3 / 2}$ & 6.9 \\
\hline \multirow[t]{3}{*}{ * } & \multirow[t]{3}{*}{$13.47 \pm 0.03$} & $21 \pm 13$ & Ne IX & 13.45 & $1 \mathrm{~s} 2 \mathrm{p}^{1} \mathrm{P}_{1} \rightarrow 1 \mathrm{~s}^{2}{ }^{1} \mathrm{~S}_{0}$ & 6.6 \\
\hline & & & Fe XIX & 13.46 & $2 \mathrm{~s}^{2} 2 \mathrm{p}^{3}\left({ }^{2} \mathrm{D}\right) 3 \mathrm{~d}^{3} \mathrm{~S}_{1} \rightarrow 2 \mathrm{~s}^{2} 2 \mathrm{p}^{4}{ }^{3} \mathrm{P}_{2}$ & 6.9 \\
\hline & & & Fe XIX & 13.42 & $2 \mathrm{~s}^{2} 2 \mathrm{p}^{3}\left({ }^{2} \mathrm{D}\right) 3 \mathrm{~d}^{1} \mathrm{~F}_{3} \rightarrow 2 \mathrm{~s}^{2} 2 \mathrm{p}^{4}{ }^{3} \mathrm{P}_{2}$ & 6.9 \\
\hline$*$ & $13.52 \pm 0.02$ & $35 \pm 14$ & Fe XIX & 13.52 & $2 \mathrm{~s}^{2} 2 \mathrm{p}^{3}\left({ }^{2} \mathrm{D}\right) 3 \mathrm{~d}^{3} \mathrm{D}_{3} \rightarrow 2 \mathrm{~s}^{2} 2 \mathrm{p}^{4}{ }^{3} \mathrm{P}_{2}$ & 6.9 \\
\hline & & & Fe XXI & 13.51 & $1 \mathrm{~s}^{2} 2 \mathrm{~s} 2 \mathrm{p}_{1 / 2}^{2} 3 \mathrm{~s} \rightarrow 1 \mathrm{~s}^{2} 2 \mathrm{~s}^{2} \mathrm{p}^{3}{ }^{3} \mathrm{D}_{1}$ & 7.0 \\
\hline & & & Fe XIX & 13.50 & $1 \mathrm{~s}^{2} 2 \mathrm{~s}^{2} 2 \mathrm{p}_{1 / 2} 2 \mathrm{p}_{3 / 2}^{2} 3 \mathrm{~d}_{3 / 2} \rightarrow 2 \mathrm{~s}^{2} 2 \mathrm{p}^{4}{ }^{3} \mathrm{P}_{2}$ & 6.9 \\
\hline & $13.69 \pm 0.02$ & $30 \pm 14$ & Ne IX & 13.70 & $1 \mathrm{~s} 2 \mathrm{~s}^{3} \mathrm{~S}_{1} \rightarrow 1 \mathrm{~s}^{2}{ }^{1} \mathrm{~S}_{0}$ & 6.6 \\
\hline & & & Fe XIX & 13.65 & $2 \mathrm{~s}^{2} 2 \mathrm{p}^{3}\left({ }^{2} \mathrm{D}\right) 3 \mathrm{~d}^{3} \mathrm{~F}_{3} \rightarrow 2 \mathrm{~s}^{2} 2 \mathrm{p}^{4}{ }^{3} \mathrm{P}_{2}$ & 6.9 \\
\hline & & & Fe XIX & 13.73 & $1 \mathrm{~s}^{2} 2 \mathrm{~s}^{2} 2 \mathrm{p}_{1 / 2} 2 \mathrm{p}_{3 / 2}^{2} 3 \mathrm{~d}_{5 / 2} \rightarrow 2 \mathrm{~s}^{2} 2 \mathrm{p}^{4}{ }^{3} \mathrm{P}_{1}$ & 6.9 \\
\hline & & & Fe XIX & 13.68 & $1 \mathrm{~s}^{2} 2 \mathrm{~s}^{2} 2 \mathrm{p}_{1 / 2} 2 \mathrm{p}_{3 / 2}^{2} 3 \mathrm{~d}_{3 / 2} \rightarrow 2 \mathrm{~s}^{2} 2 \mathrm{p}^{4}{ }^{3} \mathrm{P}_{1}$ & 6.9 \\
\hline & $13.81 \pm 0.01$ & $52 \pm 14$ & Fe XIX & 13.80 & $1 \mathrm{~s}^{2} 2 \mathrm{~s}^{2} 2 \mathrm{p}_{1 / 2} 2 \mathrm{p}_{3 / 2}^{2} 3 \mathrm{~d}_{5 / 2} \rightarrow 2 \mathrm{~s}^{2} 2 \mathrm{p}^{4}{ }^{3} \mathrm{P}_{2}$ & 6.9 \\
\hline & & & Fe XVII & 13.82 & $2 \mathrm{~s} 2 \mathrm{p}^{6} 3 \mathrm{p}^{1} \mathrm{P}_{1} \rightarrow 2 \mathrm{~s}^{2} 2 \mathrm{p}^{6}{ }^{1} \mathrm{~S}_{0}$ & 6.8 \\
\hline & & & Fe XIX & 13.76 & $2 s^{2} 2 p^{3}\left({ }^{2} D\right) 3 d^{3} S_{1} \rightarrow 2 s^{2} 2 p^{4}{ }^{1} D_{2}$ & 6.9 \\
\hline & & & $\mathrm{Fe} X \mathrm{X}$ & 13.77 & $2 s^{2} 2 p^{2}\left({ }^{3} \mathrm{P}\right) 3 s^{4} \mathrm{P}_{5 / 2} \rightarrow 2 s^{2} 2 p^{3}{ }^{4} S_{3 / 2}$ & 7.0 \\
\hline & & & Ni XIX & 13.78 & $2 \mathrm{~s}^{2} 2 \mathrm{p}^{5}\left({ }^{2} \mathrm{P}\right) 3 \mathrm{~s}^{1} \mathrm{P}_{1} \rightarrow 2 \mathrm{~s}^{2} 2 \mathrm{p}^{6}{ }^{1} \mathrm{~S}_{0}$ & 6.8 \\
\hline & $13.98 \pm 0.02$ & $40 \pm 14$ & Fe XX & 13.96 & $2 s^{2} 2 p^{2}\left({ }^{3} \mathrm{P}\right) 3 s^{4} \mathrm{P}_{1 / 2} \rightarrow 2 \mathrm{~s}^{2} 2 \mathrm{p}^{3}{ }^{4} \mathrm{~S}_{3 / 2}$ & 7.0 \\
\hline & & & Fe XVIII & 13.95 & $2 s^{2} 2 p^{4}\left({ }^{1} S\right) 3 d^{2} D_{5 / 2} \rightarrow 2 s^{2} 2 p^{5}{ }^{2} P_{3 / 2}$ & 6.9 \\
\hline & & & Fe XIX & 13.95 & $1 \mathrm{~s}^{2} 2 \mathrm{~s}^{2} 2 \mathrm{p}_{1 / 2}^{2} 2 \mathrm{p}_{3 / 2} 3 \mathrm{~d}_{3 / 2} \rightarrow 2 \mathrm{~s}^{2} 2 \mathrm{p}^{4}{ }^{3} \mathrm{P}_{2}$ & 6.9 \\
\hline & & & Fe XIX & 13.96 & $1 \mathrm{~s}^{2} 2 \mathrm{~s}^{2} 2 \mathrm{p}_{1 / 2}^{2} 2 \mathrm{p}_{3 / 2} 3 \mathrm{~d}_{3 / 2} \rightarrow 2 \mathrm{~s}^{2} 2 \mathrm{p}^{4}{ }^{3} \mathrm{P}_{2}$ & 6.9 \\
\hline & $14.05 \pm 0.01$ & $51 \pm 15$ & Fe XXI & 14.01 & $1 \mathrm{~s}^{2} 2 \mathrm{~s}^{2} 2 \mathrm{p}_{1 / 2} 3 \mathrm{p}_{1 / 2} \rightarrow 1 \mathrm{~s}^{2} 2 \mathrm{~s} 2 \mathrm{p}^{3}{ }^{3} \mathrm{D}_{1}$ & 7.0 \\
\hline & & & Ni XIX & 14.04 & $2 s^{2} 2 p^{5}\left({ }^{2} P\right) 3 s^{3} P_{1} \rightarrow 2 s^{2} 2 p^{6}{ }^{1} S_{0}$ & 6.8 \\
\hline & & & Ni XIX & 14.08 & $2 s^{2} 2 p^{5}\left({ }^{2} P\right) 3 s^{3} P_{2} \rightarrow 2 s^{2} 2 p^{6}{ }^{1} S_{0}$ & 6.8 \\
\hline & & & Fe XIX & 14.07 & $1 \mathrm{~s}^{2} 2 \mathrm{~s} 2 \mathrm{p}_{1 / 2}^{2} 2 \mathrm{p}_{3 / 2}^{2} 3 \mathrm{~d}_{3 / 2} \rightarrow 2 \mathrm{~s} 2 \mathrm{p}^{5}{ }^{3} \mathrm{P}_{2}$ & 6.9 \\
\hline
\end{tabular}


E. Franciosini et al.: XMM-Newton observation of SU Aurigae and the surrounding region, Online Material p 4

Table 2. continued.

\begin{tabular}{|c|c|c|c|c|c|c|}
\hline & $\begin{array}{l}\lambda_{\mathrm{obs}} \\
(\AA)\end{array}$ & Total counts & Ion & $\begin{array}{r}\lambda_{\text {pred }} \\
(\AA)\end{array}$ & $\begin{array}{l}\text { Transition } \\
\text { (upper } \rightarrow \text { lower) }\end{array}$ & $\log T_{\max }$ \\
\hline \multirow[t]{14}{*}{ * } & \multirow[t]{4}{*}{$14.22 \pm 0.01$} & \multirow[t]{4}{*}{$92 \pm 17$} & Fe XVIII & 14.21 & $1 \mathrm{~s}^{2} 2 \mathrm{~s}^{2} 2 \mathrm{p}_{1 / 2} 2 \mathrm{p}_{3 / 2}^{3} 3 \mathrm{~d}_{5 / 2} \rightarrow 2 \mathrm{~s}^{2} 2 \mathrm{p}^{5}{ }^{2} \mathrm{P}_{3 / 2}$ & 6.9 \\
\hline & & & Fe XVIII & 14.21 & $2 \mathrm{~s}^{2} 2 \mathrm{p}^{4}\left({ }^{1} \mathrm{D}\right) 3 \mathrm{~d}^{2} \mathrm{D}_{5 / 2} \rightarrow 2 \mathrm{~s}^{2} 2 \mathrm{p}^{5}{ }^{2} \mathrm{P}_{3 / 2}$ & 6.9 \\
\hline & & & Fe XX & 14.27 & $1 \mathrm{~s}^{2} 2 \mathrm{~s} 2 \mathrm{p}_{1 / 2}^{2} 2 \mathrm{p}_{3 / 2} 3 \mathrm{~s} \rightarrow 2 \mathrm{~s} 2 \mathrm{p}^{4}{ }^{4} \mathrm{P}_{5 / 2}$ & 7.0 \\
\hline & & & Fe XVIII & 14.26 & $2 s^{2} 2 p^{4}\left({ }^{1} D\right) 3 d^{2} S_{1 / 2} \rightarrow 2 s^{2} 2 p^{5}{ }^{2} P_{3 / 2}$ & 6.9 \\
\hline & \multirow[t]{3}{*}{$14.35 \pm 0.02$} & \multirow[t]{3}{*}{$42 \pm 15$} & Fe XVIII & 14.34 & $2 \mathrm{~s}^{2} 2 \mathrm{p}^{4}\left({ }^{1} \mathrm{D}\right) 3 \mathrm{~d}^{2} \mathrm{P}_{1 / 2} \rightarrow 2 \mathrm{~s}^{2} 2 \mathrm{p}^{5}{ }^{2} \mathrm{P}_{1 / 2}$ & 6.9 \\
\hline & & & Fe XVIII & 14.34 & $2 \mathrm{~s}^{2} 2 \mathrm{p}^{4}\left({ }^{1} \mathrm{D}\right) 3 \mathrm{~d}^{2} \mathrm{D}_{3 / 2} \rightarrow 2 \mathrm{~s}^{2} 2 \mathrm{p}^{5}{ }^{2} \mathrm{P}_{1 / 2}$ & 6.9 \\
\hline & & & Fe XX & 14.33 & $1 \mathrm{~s}^{2} 2 \mathrm{~s}^{2} 2 \mathrm{p}_{3 / 2}^{2} 3 \mathrm{p}_{3 / 2} \rightarrow 2 \mathrm{~s} 2 \mathrm{p}^{4}{ }^{4} \mathrm{P}_{5 / 2}$ & 7.0 \\
\hline & \multirow[t]{3}{*}{$14.42 \pm 0.02$} & \multirow[t]{3}{*}{$26 \pm 14$} & Fe XVIII & 14.37 & $2 s^{2} 2 p^{4}\left({ }^{3} \mathrm{P}\right) 3 \mathrm{~d}^{2} \mathrm{D}_{5 / 2} \rightarrow 2 \mathrm{~s}^{2} 2 \mathrm{p}^{5}{ }^{2} \mathrm{P}_{3 / 2}$ & 6.9 \\
\hline & & & Fe $x x$ & 14.42 & $1 \mathrm{~s}^{2} 2 \mathrm{~s} 2 \mathrm{p}_{1 / 2}^{2} 2 \mathrm{p}_{3 / 2} 3 \mathrm{~s} \rightarrow 2 \mathrm{~s} 2 \mathrm{p}^{4}{ }^{4} \mathrm{P}_{3 / 2}$ & 7.0 \\
\hline & & & Fe XVIII & 14.43 & $1 \mathrm{~s}^{2} 2 \mathrm{~s}^{2} 2 \mathrm{p}_{1 / 2} 2 \mathrm{p}_{3 / 2}^{3} 3 \mathrm{~d}_{5 / 2} \rightarrow 2 \mathrm{~s}^{2} 2 \mathrm{p}^{5}{ }^{2} \mathrm{P}_{1 / 2}$ & 6.9 \\
\hline & \multirow[t]{3}{*}{$14.53 \pm 0.02$} & \multirow[t]{3}{*}{$39 \pm 14$} & Fe XVIII & 14.53 & $2 \mathrm{~s}^{2} 2 \mathrm{p}^{4}\left({ }^{3} \mathrm{P}\right) 3 \mathrm{~d}^{2} \mathrm{~F}_{5 / 2} \rightarrow 2 \mathrm{~s}^{2} 2 \mathrm{p}^{5}{ }^{2} \mathrm{P}_{3 / 2}$ & 6.9 \\
\hline & & & Fe XVIII & 14.57 & $2 \mathrm{~s}^{2} 2 \mathrm{p}^{4}\left({ }^{3} \mathrm{P}\right) 3 \mathrm{~d}^{4} \mathrm{P}_{3 / 2} \rightarrow 2 \mathrm{~s}^{2} 2 \mathrm{p}^{5}{ }^{2} \mathrm{P}_{3 / 2}$ & 6.9 \\
\hline & & & Fe XVIII & 14.51 & $1 \mathrm{~s}^{2} 2 \mathrm{~s}^{2} 2 \mathrm{p}_{1 / 2}^{2} 2 \mathrm{p}_{3 / 2}^{2} 3 \mathrm{~d}_{3 / 2} \rightarrow 2 \mathrm{~s}^{2} 2 \mathrm{p}^{52} \mathrm{P}_{3 / 2}$ & 6.8 \\
\hline & \multirow[t]{2}{*}{$14.64 \pm 0.02$} & \multirow[t]{2}{*}{$36 \pm 14$} & Fe XIX & 14.66 & $2 s^{2} 2 p^{3}\left({ }^{2} D\right) 3 s^{3} D_{3} \rightarrow 2 s^{2} 2 p^{4}{ }^{3} P_{2}$ & 6.9 \\
\hline & & & Fe XVIII & 14.62 & $2 \mathrm{~s}^{2} 2 \mathrm{p}^{4}\left({ }^{3} \mathrm{P}\right) 3 \mathrm{~d}^{2} \mathrm{P}_{3 / 2} \rightarrow 2 \mathrm{~s}^{2} 2 \mathrm{p}^{5}{ }^{2} \mathrm{P}_{1 / 2}$ & 6.8 \\
\hline \multirow[t]{4}{*}{$*$} & \multirow[t]{4}{*}{$14.93 \pm 0.02$} & \multirow[t]{4}{*}{$26 \pm 15$} & Fe XIX & 14.96 & $2 s^{2} 2 p^{3}\left({ }^{4} S\right) 3 s^{3} S_{1} \rightarrow 2 s^{2} 2 p^{4}{ }^{3} P_{2}$ & 6.9 \\
\hline & & & $\mathrm{Fe} X \mathrm{X}$ & 14.92 & $1 \mathrm{~s}^{2} 2 \mathrm{~s}^{2} 2 \mathrm{p}_{1 / 2} 2 \mathrm{p}_{3 / 2} 3 \mathrm{p}_{3 / 2} \rightarrow 2 \mathrm{~s} 2 \mathrm{p}^{4}{ }^{4} \mathrm{P}_{3 / 2}$ & 7.0 \\
\hline & & & Fe XX & 14.97 & $1 \mathrm{~s}^{2} 2 \mathrm{~s}^{2} 2 \mathrm{p}_{1 / 2} 2 \mathrm{p}_{3 / 2} 3 \mathrm{p}_{3 / 2} \rightarrow 2 \mathrm{~s} 2 \mathrm{p}^{4}{ }^{4} \mathrm{P}_{1 / 2}$ & 7.0 \\
\hline & & & Fe XX & 14.95 & $1 \mathrm{~s}^{2} 2 \mathrm{~s}^{2} 2 \mathrm{p}_{1 / 2} 2 \mathrm{p}_{3 / 2} 3 \mathrm{p}_{1 / 2} \rightarrow 2 \mathrm{~s} 2 \mathrm{p}^{4}{ }^{4} \mathrm{P}_{5 / 2}$ & 7.0 \\
\hline \multirow[t]{2}{*}{ * } & \multirow[t]{2}{*}{$15.03 \pm 0.01$} & \multirow[t]{2}{*}{$152 \pm 19$} & Fe XVII & 15.01 & $2 \mathrm{~s}^{2} 2 \mathrm{p}^{5}\left({ }^{2} \mathrm{P}\right) 3 \mathrm{~d}^{1} \mathrm{P}_{1} \rightarrow 2 \mathrm{~s}^{2} 2 \mathrm{p}^{6}{ }^{1} \mathrm{~S}_{0}$ & 6.7 \\
\hline & & & Fe XIX & 15.08 & $2 s^{2} 2 p^{3}\left({ }^{4} S\right) 3 s^{5} S_{2} \rightarrow 2 s^{2} 2 p^{4}{ }^{3} P_{2}$ & 6.9 \\
\hline \multirow[t]{6}{*}{ * } & \multirow[t]{3}{*}{$15.20 \pm 0.01$} & \multirow[t]{3}{*}{$32 \pm 14$} & Fe XIX & 15.20 & $1 s^{2} 2 s 2 p_{1 / 2}^{2} 2 p_{3 / 2}^{2} 3 s \rightarrow 2 s 2 p^{5}{ }^{3} P_{2}$ & 6.9 \\
\hline & & & O VIII & 15.18 & $4 \mathrm{p}^{2} \mathrm{P}_{1 / 2} \rightarrow 1 \mathrm{~s}{ }^{2} \mathrm{~S}_{1 / 2}$ & 6.5 \\
\hline & & & O VIII & 15.18 & $4 \mathrm{p}^{2} \mathrm{P}_{3 / 2} \rightarrow 1 \mathrm{~s}^{2} \mathrm{~S}_{1 / 2}$ & 6.5 \\
\hline & $15.28 \pm 0.01$ & $40 \pm 14$ & Fe XVII & 15.26 & $2 s^{2} 2 p^{5}\left({ }^{2} P\right) 3 d^{3} D_{1} \rightarrow 2 s^{2} 2 p^{6}{ }^{1} S_{0}$ & 6.7 \\
\hline & \multirow[t]{2}{*}{$15.45 \pm 0.01$} & \multirow[t]{2}{*}{$35 \pm 13$} & Fe XVII & 15.45 & $2 \mathrm{~s}^{2} 2 \mathrm{p}^{5}\left({ }^{2} \mathrm{P}\right) 3 \mathrm{~d}^{3} \mathrm{P}_{1} \rightarrow 2 \mathrm{~s}^{2} 2 \mathrm{p}^{6}{ }^{1} \mathrm{~S}_{0}$ & 6.7 \\
\hline & & & Fe XIX & 15.47 & $1 s^{2} 2 s 2 p_{1 / 2}^{2} 2 p_{3 / 2}^{2} 3 s \rightarrow 2 s 2 p^{5}{ }^{3} P_{2}$ & 6.9 \\
\hline * & $15.61 \pm 0.02$ & $26 \pm 12$ & Fe XVIII & 15.62 & $2 s^{2} 2 p^{4}\left({ }^{1} D\right) 3 s^{2} D_{5 / 2} \rightarrow 2 s^{2} 2 p^{5}{ }^{2} P_{3 / 2}$ & 6.8 \\
\hline \multirow[t]{3}{*}{ * } & \multirow[t]{3}{*}{$16.02 \pm 0.01$} & \multirow[t]{3}{*}{$56 \pm 15$} & O VIII & 16.01 & $3 \mathrm{p}^{2} \mathrm{P}_{1 / 2} \rightarrow 1 \mathrm{~s}^{2} \mathrm{~S}_{1 / 2}$ & 6.5 \\
\hline & & & O VIII & 16.01 & $3 \mathrm{p}^{2} \mathrm{P}_{3 / 2} \rightarrow 1 \mathrm{~s}^{2} \mathrm{~S}_{1 / 2}$ & 6.5 \\
\hline & & & Fe XVIII & 16.00 & $2 \mathrm{~s}^{2} 2 \mathrm{p}^{4}\left({ }^{3} \mathrm{P}\right) 3 \mathrm{~s}^{2} \mathrm{P}_{3 / 2} \rightarrow 2 \mathrm{~s}^{2} 2 \mathrm{p}^{5}{ }^{2} \mathrm{P}_{3 / 2}$ & 6.8 \\
\hline * & $16.10 \pm 0.02$ & $44 \pm 14$ & Fe XVIII & 16.07 & $2 s^{2} 2 p^{4}\left({ }^{3} \mathrm{P}\right) 3 s^{4} \mathrm{P}_{5 / 2} \rightarrow 2 \mathrm{~s}^{2} 2 \mathrm{p}^{5}{ }^{2} \mathrm{P}_{3 / 2}$ & 6.8 \\
\hline & & & Fe XIX & 16.11 & $21 s^{2} 2 s^{2} 2 p_{1 / 2} 2 p_{3 / 2}^{2} 3 p_{1 / 2} \rightarrow 2 s 2 p^{5}{ }^{3} P_{2}$ & 6.9 \\
\hline & $16.33 \pm 0.02$ & $22 \pm 11$ & Fe XVIII & 16.32 & $2 \mathrm{~s} 2 \mathrm{p}^{5} 3 \mathrm{~s}^{2} \mathrm{P}_{3 / 2} \rightarrow 2 \mathrm{~s} 2 \mathrm{p}^{6}{ }^{2} \mathrm{~S}_{1 / 2}$ & 6.8 \\
\hline & & & Fe XVII & 16.35 & $2 \mathrm{~s}^{2} 2 \mathrm{p}^{5}\left({ }^{2} \mathrm{P}\right) 3 \mathrm{p}^{3} \mathrm{D}_{2} \rightarrow 2 \mathrm{~s}^{2} 2 \mathrm{p}^{6}{ }^{1} \mathrm{~S}_{0}$ & 6.7 \\
\hline & & & Fe XIX & 16.34 & $1 \mathrm{~s}^{2} 2 \mathrm{~s}^{2} 2 \mathrm{p}_{1 / 2} 2 \mathrm{p}_{3 / 2}^{2} 3 \mathrm{p}_{1 / 2} \rightarrow 2 \mathrm{~s} 2 \mathrm{p}^{5}{ }^{3} \mathrm{P}_{2}$ & 6.9 \\
\hline * & $16.78 \pm 0.01$ & $60 \pm 13$ & Fe XVII & 16.78 & $2 \mathrm{~s}^{2} 2 \mathrm{p}^{5}\left({ }^{2} \mathrm{P}\right) 3 \mathrm{~s}^{1} \mathrm{P}_{1} \rightarrow 2 \mathrm{~s}^{2} 2 \mathrm{p}^{6}{ }^{1} \mathrm{~S}_{0}$ & 6.7 \\
\hline$*$ & $17.06 \pm 0.01$ & $73 \pm 14$ & Fe XVII & 17.05 & $2 \mathrm{~s}^{2} 2 \mathrm{p}^{5}\left({ }^{2} \mathrm{P}\right) 3 \mathrm{~s}^{3} \mathrm{P}_{1} \rightarrow 2 \mathrm{~s}^{2} 2 \mathrm{p}^{6}{ }^{1} \mathrm{~S}_{0}$ & 6.7 \\
\hline & & & Fe XVII & 17.10 & $2 \mathrm{~s}^{2} 2 \mathrm{p}^{5}\left({ }^{2} \mathrm{P}\right) 3 \mathrm{~s}^{3} \mathrm{P}_{2} \rightarrow 2 \mathrm{~s}^{2} 2 \mathrm{p}^{6}{ }^{1} \mathrm{~S}_{0}$ & 6.7 \\
\hline * & $18.99 \pm 0.01$ & $66 \pm 14$ & O VIII & 18.97 & $2 \mathrm{p}^{2} \mathrm{P}_{1 / 2} \rightarrow 1 \mathrm{~s}^{2} \mathrm{~S}_{1 / 2}$ & 6.5 \\
\hline & & & O VIII & 18.97 & $2 \mathrm{p}^{2} \mathrm{P}_{3 / 2} \rightarrow 1 \mathrm{~s}^{2} \mathrm{~S}_{1 / 2}$ & 6.5 \\
\hline
\end{tabular}

